\title{
7 \\ Tests expérimentaux de la non localifé quantique avec des paires de photons intriqués
}

\author{
Alain Aspect \\ Institut d'Optique Graduate School, Université Paris-Saclay, \\ 2 avenue Augustin Fresnel, 91128 Palaiseau Cedex - France
}

\section{Résumé}

Depuis leur découverte par Einstein, Podolsky et Rosen en 1935, les états quantiques intriqués n'ont pas cessé de susciter l'intérêt des physiciens. D'abord simples sujets de réflexion sur les fondements de la mécanique quantique, ils sont devenus, après les travaux de John Bell, l'enjeu d'expériences qui ont confirmé la non séparabilité quantique : dans un état intriqué, deux particules, même très éloignées l'une de l'autre, ne peuvent être décrites comme des entités séparées ayant des propriétés individualisées. En parallèle avec l'approfondissement de la réflexion sur la réalité physique et notre représentation du monde, ces travaux débouchent aussi, de façon surprenante, sur des possibilités d'application : cryptographie quantique, téléportation quantique, ordinateur quantique.

\section{Abstract}

Since their discovery by Einstein, Podolsky and Rosen in 1935, the entangled quantum states have not ceased to arouse the interest of physicists. First, simple subjects of reflection on the foundations of quantum mechanics, they became, after John Bell's work, the subjects of experiments that confirmed quantum non-separability: in an entangled state, two particles, even very far apart, cannot be described as separate entities with individualized properties. In parallel with the deepening of the reflection on physical reality and our representation of the world, these works also lead, surprisingly, to possibilities of application: quantum cryptography, quantum teleportation, quantum computer. 


\begin{abstract}
Note
Ce texte est la version écrite de la conférence du même nom donnée en 1999 dans le cadre de la cinquième rencontre « Physique et interrogations fondamentales » et publiée dans L'élémentaire \& le complexe, édité par le regretté Michel Crozon \& Yves Sacquin', EDP-Sciences (200I).
\end{abstract}

\title{
I. Introduction
}

Le problème de l'interprétation du formalisme quantique, et en particulier de son caractère probabiliste, a donné lieu entre Bohr et Einstein à un débat commencé à la fin des années 1920, et auquel seule la disparition des protagonistes mettra un terme au début des années 1950. Un moment décisif de ce débat fut la publication en 1935 du célèbre article EPR (Einstein, Podolsky, et Rosen) [I], où fut présenté un état « intriqué » de deux particules éloignées dans l'espace. Einstein nota immédiatement l'étrangeté, à l'aune des concepts habituels issus de la physique classique, des corrélations prévues par la physique quantique dans cette situation. II pensa pouvoir en déduire que le formalisme quantique devait être complété, ce qui remettait en cause «l'interprétation de Copenhague », et qui fut véhémentement contesté par Bohr. Cette question focalisa l'essentiel du débat ultérieur entre les deux physiciens. Notons d'emblée qu'Einstein ne contestait nullement la correction des prédictions du formalisme quantique : la controverse portait sur l'interprétation de ce formalisme.

La situation fut radicalement bouleversée en 1965, lorsque John Bell découvrit que si l'on suit jusqu'au bout les idées d'Einstein, on aboutit - dans certaines situations très rares du type EPR - à une contradiction quantitative avec les prédictions quantiques [2]. Le débat initialement de nature épistémologique devenait une question scientifique, susceptible d'être tranchée expérimentalement. De telles expériences allaient effectivement être menées à partir des années 1970 .

La première partie de cette présentation (parties 2 à 6), vise à expliquer ce que sont le théorème de Bell et les inégalités de Bell, et pourquoi je les trouve si importants. Elle est suivie d'une brève présentation des expériences de première génération, conduites entre 1971 et 1976. Puis je décrirai plus en détail les trois expériences de deuxième génération, réalisées à Orsay entre 1976 et 1982, avec des paires de photons corrélés produites dans des cascades radiatives atomiques excitées par laser. Je présenterai enfin les expériences de troisième génération, développées à partir de la fin des années 1980 : utilisant des sources encore plus efficaces, basées sur la division paramétrique de photons laser, elles ont conduit récemment à des tests des inégalités de Bell encore plus proches du schéma idéal. Permettant l'injection des paires de photons EPR corrélés dans des fibres optiques, ces sources ont par ailleurs ouvert la voie au domaine de la cryptographie quantique et de l'information quantique.

(I) L'AEIS remercie Yves Sacquin pour son aimable autorisation à reproduire cet article 


\section{Pourquoi des paramètres supplémentaires ? L'expérience de pensée d'Einstein- Podolsky-Rosen-Bohm}

\section{I Schéma expérimental}

Considérons la variante de Bohm[3] de l'expérience de pensée EPR (figure I). Une source $S$ émet une paire de photons $v_{1}$ and $v_{2}$, séloignant dans des directions opposées suivant $O z$. Supposons que la partie polarisation du vecteur d'état décrivant la paire puisse s'écrire :

$$
\left|\Psi\left(v_{1}, v_{2}\right)\right\rangle=\frac{1}{\sqrt{2}}\{|x, x\rangle+|y, y\rangle\}
$$

où $|x\rangle$ et $|y\rangle$ sont des états de polarisation linéaire. Cet état est remarquable. Il est impossible de le factoriser en un produit tensoriel de deux états de polarisation définie pour chaque photon. On ne peut donc attribuer de polarisation à chaque photon pris séparément.

Effectuons une mesure de polarisation linéaire sur chacun de ces photons, avec les analyseurs $I$ and $I I$. L'analyseur $I$, dans l'orientation caractérisée par le vecteur unitaire a, est suivi de deux détecteurs donnant les résultats + ou - , correspondant à une polarisation linéaire parallèle ou perpendiculaire à a pour le photon $v_{1}$. L'analyseur II, dans l'orientation b, agit de façon analogue sur le photon $v_{2}{ }^{2}$.

Un calcul simple de mécanique quantique fournit les résultats prévus pour ces mesures de polarisation, simples ou en coïncidences. Nous noterons $P_{ \pm}(\mathbf{a})$ la probabilité (simple) d'obtenir le résultat \pm sur le photon $v_{1}$; de même $P_{ \pm}(\mathbf{b})$ caractérise le résultat de la mesure de polarisation sur le photon $v_{2}$. La prédiction quantique s'écrit :

$$
\begin{aligned}
& P_{+}(\mathbf{a})=P_{-}(\mathbf{a})=1 / 2 \\
& P_{+}(\mathbf{b})=P_{-}(\mathbf{b})=1 / 2
\end{aligned}
$$

Ces résultats sont en accord avec l'observation qu'on ne peut attribuer aucune polarisation particulière à chacun des photons, de telle sorte qu'une mesure individuelle de polarisation donne un résultat aléatoire.

Nous nous intéressons à présent aux probabilités $P_{ \pm \pm}(\mathbf{a}, \mathbf{b})$ de détection conjointe de $v_{1}$ et $v_{2}$ dans les canaux + ou - des polariseurs $I$ ou $I I$, orientés suivant a et $\mathbf{b}$. La prédiction quantique s'écrit :

(2) Cette situation est en correspondance biunivoque avec l'expérience de pensée EPR Bohm portant sur une paire de spins 1/2 dans un état singulet, sur lesquels des filtres de Stern-Gerlach orientables effectuent des mesures de composantes de moment cinétique. 


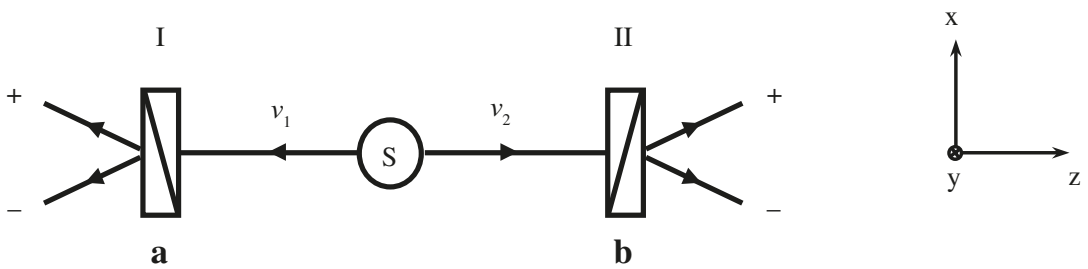

Figure 1. Expérience de pensée d'Einstein-Podolsky-Bohm avec des photons. Les deux photons $v_{1}$ and $\nu_{2}$,émis dans l'état de polarisation $|\Psi(1,2)\rangle$ de l'équation (1), sont analysés par des polariseurs linéaires I et II orientés suivant $\mathbf{a}$ et $\mathbf{b}$. On peut mesurer les probabilités de détection simples ou conjointes dans les voies de sortie des polariseurs.

$$
\begin{aligned}
& P_{++}(\mathbf{a}, \mathbf{b})=P_{--}(\mathbf{a}, \mathbf{b})=\frac{1}{2} \cos ^{2}(\mathbf{a}, \mathbf{b}) \\
& P_{+-}(\mathbf{a}, \mathbf{b})=P_{-+}(\mathbf{a}, \mathbf{b})=\frac{1}{2} \sin ^{2}(\mathbf{a}, \mathbf{b})
\end{aligned}
$$

Nous allons maintenant montrer que ces prédictions ont des conséquences de très grande portée.

\subsection{Corrélations}

Considérons d'abord la situation particulière $(\mathbf{a}, \mathbf{b})=0$, où les deux polariseurs sont parallèles. La prédiction quantique pour les probabilités conjointes (équations 3 ) s'écrit :

$$
\begin{aligned}
& P_{++}(\mathbf{a}, \mathbf{a})=P_{--}(\mathbf{a}, \mathbf{a})=\frac{1}{2} \\
& P_{+-}(\mathbf{a}, \mathbf{a})=P_{-+}(\mathbf{a}, \mathbf{a})=0
\end{aligned}
$$

D'après ce résultat, lorsque le photon $v_{1}$ est trouvé dans le canal + du polariseur $I$, alors on est certain de trouver $v_{2}$ dans le canal + de $I I$ (et de même pour les résultats -). Ainsi, pour des polariseurs parallèles, les résultats de mesure de polarisation sur $v_{1}$ et $v_{2}$ sont totalement corrélés.

Pour caractériser quantitativement le degré de corrélation entre quantités aléatoires, on utilise le coefficient de corrélation. Pour les mesures de polarisation considérées ici, il s'écrit :

$$
E(\mathbf{a}, \mathbf{b})=P_{++}(\mathbf{a}, \mathbf{b})+P_{--}(\mathbf{a}, \mathbf{b})-P_{+-}(\mathbf{a}, \mathbf{b})-P_{-+}(\mathbf{a}, \mathbf{b})
$$

En reportant dans cette définition les prédictions quantiques (3), on obtient un coefficient de corrélation de polarisation

$$
E_{Q M}(\mathbf{a}, \mathbf{b})=\cos 2(\mathbf{a}, \mathbf{b})
$$


Pour le cas particulier des polariseurs parallèles $((\mathbf{a}, \mathbf{b})=0)$, on trouve $E_{\mathrm{QM}}(0)=1$, ce qui confirme que la corrélation est totale.

En conclusion, les prédictions quantiques suggèrent que, malgré le caractère apparemment aléatoire des résultats individuels, il y a une corrélation entre ces résulats aléatoires. Cette corrélation est caractérisée par l'équation (6). Pour des polariseurs parallèles (ou perpendiculaires), la corrélation est totale $\left(\left|E_{Q M}\right|=1\right)$.

\subsection{Difficultés pour construire une image à partir du formalisme quantique}

Nous pouvons chercher à trouver une image simple pour comprendre ces corrélations fortes. L'idée la plus naturelle est de suivre le calcul quantique conduisant à (3). En fait, il existe plusieurs moyens de faire ce calcul. L'un des plus directs consiste à projeter le vecteur d'état $(I)$ sur le vecteur propre associé à chaque mesure conjointe particulière. On obtient ainsi immédiatement les résultats (3). Malheureusement, ces calculs portant sur des vecteurs d'états décrivant globalement les 2 photons dans un espace abstrait, il est difficile d'en extraire une image dans notre espace ordinaire.

Pour décrire séparément les deux mesures aux deux extrémités opposées de l'expérience, nous décomposons la mesure conjointe en deux étapes. Supposons par exemple que la mesure sur le photon $v_{\text {, }}$ soit faite en premier, et donne le résultat + , le polariseur $I$ étant orienté suivant a que nous prenons parallèle à l'axe $O x$ pour simplifer $\left(a=\mathbf{e}_{x}\right)$. Ce résultat + a une probabilité de $\mathrm{I} / 2$. Pour continuer le calcul, nous devons utiliser le postulat de réduction du paquet d'onde : à la suite de cette première mesure, le nouveau vecteur d'état $\left|\Psi '\left(v_{1}, v_{2}\right)\right\rangle$ décrivant la paire est obtenu par projection du vecteur d'état initial $\left|\Psi\left(v_{1}, v_{2}\right)\right\rangle$ (équation I) sur l'espace propre associé au résultat + : cet espace à deux dimensions a pour base $\{|x, x\rangle,|x, y\rangle\}$. En utilisant le projecteur correspondant, on obtient

$$
\left|\Psi^{\prime}\left(v_{1}, v_{2}\right)\right\rangle=|x, x\rangle
$$

Cela veut dire qu'immédiatement après la première mesure, le photon $v_{1}$ prend la polarisation $|x\rangle$ : ceci paraît évident puisque la mesure, faite avec un polariseur orienté suivant $O x$, a donné le résultat + . De façon plus étonnante, le photon éloigné $v_{2}$, qui n’a encore interagi avec aucun polariseur, a lui aussi été projeté dans l'état $|x\rangle$, de polarisation bien définie, parallèle à celle trouvée pour $v_{1}$. Cette conclusion surprenante conduit bien au résultat correct (3), puisqu'une application directe de la loi de Malus montre qu'une mesure ultérieure sur le photon $v_{2}$ donnera

$$
P_{++}\left(\mathbf{e}_{x}, \mathbf{b}\right)=\frac{1}{2} \cos ^{2}\left(\mathbf{e}_{x}, \mathbf{b}\right)
$$


(le facteur I/2 correspond à la probabilité I/2 d'avoir obtenu + lors de la première mesure sur le photon $v_{1}$ ).

Le calcul en deux étapes donne donc les mêmes résultats que le calcul direct. Mais il suggère en plus une image en deux étapes, que l'on peut maintenant décrire dans l'espace réel:

I. Le photon $v_{1}$, qui n'avait pas de polarisation bien définie avant sa mesure au polariseur I, prend la polarisation associée au résultat obtenu, au moment de cette mesure : ceci n'est en rien surprenant.

2. Lorsque la mesure est effectuée sur $v_{1}$, le photon $v_{2}$, qui n'avait pas de polarisation définie avant cette mesure, est projeté dans un état de polarisation parallèle au résultat de la mesure effectuée sur $v_{1}$. Ceci est très étonnant, puisque ce brusque changement dans la description du photon $v_{2}$ se produit instantanément, quelle que soit la distance entre les photons $v_{1}$ et $v_{2}$ au moment de la mesure sur $v_{1}$.

Cette image apparaît en contradiction avec la relativité. Suivant Einstein, ce qui se passe dans une région donnée de l'espace temps ne peut pas être influencé par un événement se produisant dans une autre région de l'espace temps séparée de la première par un intervalle du genre espace (aucun signal se propageant à une vitesse inférieure ou égale à celle de la lumière ne peut connecter les deux évènements). II n'est donc pas surprenant que l'on ait essayé de trouver des images plus acceptables pour décrire les corrélations EPR. Nous allons présenter une telle image.

\subsection{Paramètres supplémentaires}

II est courant dans le monde classique d'observer des corrélations entre mesures distantes, sur deux systèmes séparés qui avaient interagi dans le passé. Par exemple, si un système mécanique de moment cinétique total nul se fragmente en deux sous l'effet d'une répulsion interne, les moments cinétiques des deux fragments resteront exactement opposés à tout instant ultérieur, en l'absence de force externe. Plus généralement, même en présence de forces, les deux moments cinétiques resteront corrélés, puisque leurs valeurs à tout instant sont déterminées par les valeurs initiales qui étaient exactement opposées.

Il est tentant d'utiliser une telle image classique pour rendre compte des corrélations $E P R$, en terme de propriété commune aux deux systèmes. Considérons à nouveau la corrélation de polarisation totale entre les mesures effectuées avec des polariseurs parallèles, $(\mathbf{a}, \mathbf{b})=0$. Quand nous trouvons + pour $v_{1}$, nous sommes sûrs de trouver aussi + pour $v_{2}$. Nous sommes alors conduits à admettre qu'il existe une propriété (Einstein parlait d'« élément de réalité physique ») appartenant à cette paire avant les mesures, et déterminant le résultat ++. Pour une autre paire, conduisant au résultat -- nous pouvons de façon analogue invoquer une propriété commune préalable, déterminant le résultat. Il suffit alors d'admettre que la moitié des paires sont émises avec la propriété ++, et la moitié avec la propriété --, pour reproduire tous les résultats obtenus dans cette configuration. 
II faut remarquer ici que de telles propriétés, différentes d'une paire à l'autre, ne sont pas prises en compte par le vecteur d'état quantique $\left|\Psi\left(v_{1}, v_{2}\right)\right\rangle$ qui est le même pour toutes les paires. C'est pour cette raison, qu'Einstein en tira la conclusion que la mécanique quantique n'est pas complète. Et c'est pourquoi de telles propriétés supplémentaires sont désignées par le terme de « paramètres supplémentaires », ou encore « variables cachées ».

En conclusion, il semble possible de « comprendre » les corrélations EPR par une image de type classique, impliquant des paramètres supplémentaires différents d'une paire à l'autre. On peut espérer retrouver les prédictions statistiques de la mécanique quantique, lorsqu'on moyenne sur les paramètres supplémentaires. Il semble que telle était la position d'Einstein $[4,5,6]$. Notons qu'à cette étape du raisonnement, une telle position n'est pas en contradiction avec la mécanique quantique : il n'y a aucun problème logique à admettre pleinement les prédictions quantiques, tout en invoquant des paramètres supplémentaires donnant une image acceptable des corrélations EPR.

\section{Inégalités de Bell}

\section{I Formalisme}

Bell traduisit en termes mathématiques les conséquences de la discussion ci-dessus, et il introduisit explicitement des paramètres supplémentaires, notés $\lambda$. La distribution de ces paramètres sur un ensemble de paires émises est spécifiée par une densité de probabilité $\rho(\lambda)$, telle que :

$$
\begin{aligned}
& \rho(\lambda) \geq 0 \\
& \int d \lambda \rho(\lambda)=1
\end{aligned}
$$

Pour une paire donnée, caractérisée par un paramètre supplémentaire $\lambda$, les résultats de mesures sont donnés par des fonctions bivaluées

$$
\begin{array}{ll}
A(\lambda, \mathbf{a})= \pm 1 & \text { au polariseur I (orientation } \mathbf{a}) \\
B(\lambda, \mathbf{b})= \pm 1 & \text { au polariseur II (orientation } \mathbf{b} \text { ) }
\end{array}
$$

Une Théorie à Paramètre Supplémentaire particulière est complètement définie par la forme explicite des fonctions $\rho(\lambda), A(\lambda, \mathbf{a})$ et $B(\lambda, \mathbf{b})$. Il est alors facile d'exprimer les probalités des divers résultats de mesures. Par exemple, nous remarquons que la fonction $1 / 2[A(\lambda, \mathbf{a})+1]$ prend la valeur + I pour le résultat + , et 0 pour le résultat - (et de façon similaire $1 / 2[1-B(\lambda, \mathbf{b})]$ prend la valeur + pour le résultat - et 0 pour le résultat + ), nous pouvons en déduire

$$
\begin{aligned}
& P_{+}(\mathrm{a})=\int d \lambda \rho(\lambda) \frac{[A(\lambda, \mathrm{a})+1]}{2} \\
& P_{+-}(\mathrm{a}, \mathrm{b})=\int d \lambda \rho(\lambda) \frac{[A(\lambda, \mathrm{a})+1]}{2} \frac{[1-B(\lambda, \mathrm{b})]}{2}
\end{aligned}
$$


Finalement, la fonction de corrélation définie à l'équation (5) prend dans un tel formalisme la forme particulièrement simple

$$
E(\mathbf{a}, \mathbf{b})=\int d \lambda \rho(\lambda) A(\lambda, \mathbf{a}) B(\lambda, \mathbf{b})
$$

\subsection{Un exemple (naiif) de théorie à paramètres supplémentaires}

Comme exemple de Théorie à Paramètre Supplémentaire, nous présentons un modèle où chaque photon qui se propage le long de l'axe $O z$ est supposé posséder une polarisation linéaire bien définie, déterminée par son angle $\left(\lambda_{1}\right.$ ou $\left.\lambda_{2}\right)$ avec l'axe $x$. Afin de rendre compte de la forte corrélation, nous supposons que les deux photons d'une même paire sont émis avec la même polarisation linéaire, définie par un angle commun $\lambda$ (figure 2).

La polarisation des diverses paires est distribuée aléatoirement, ce que nous décrivons par une densité de probabilité isotrope $\rho(\lambda)$ :

$$
\rho(\lambda)=\frac{1}{2 \pi}
$$

Pour compléter notre modèle, nous devons donner une forme explicite pour les fonctions $A(\lambda, \mathbf{a})$ et $B(\lambda, \mathbf{b})$. Nous prenons la forme suivante

$$
\begin{aligned}
& A(\lambda, \mathbf{a})=\text { signe }\left\{\cos 2\left(\theta_{I}-\lambda\right)\right\} \\
& B(\lambda, \mathbf{b})=\text { signe }\left\{\cos 2\left(\theta_{I I}-\lambda\right)\right\}
\end{aligned}
$$

où les angles $\theta_{I}$ et $\theta_{I I}$ indiquent les orientations des polariseurs. Remarquons que ces formes sont tout à fait raisonnables. La fonction $A(\lambda$,a) prend la valeur $+\mid$ quand la polarisation du photon $v_{1}$ fait un angle de moins que $\pi / 4$ avec la direction d'analyse $\mathbf{a}$, et la valeur -I pour le cas complémentaire (la polarisation est alors plus proche de la perpendiculaire à a).

Avec ce modèle explicite, nous pouvons utiliser les équations (II) pour calculer les probabilités de diverses mesures. Nous trouvons par exemple des probabilités simples (I5) identiques aux prédictions quantiques:

$$
P_{+}(\mathbf{a})=P_{-}(\mathbf{a})=P_{+}(\mathbf{b})=P_{-}(\mathbf{b})=\frac{1}{2}
$$




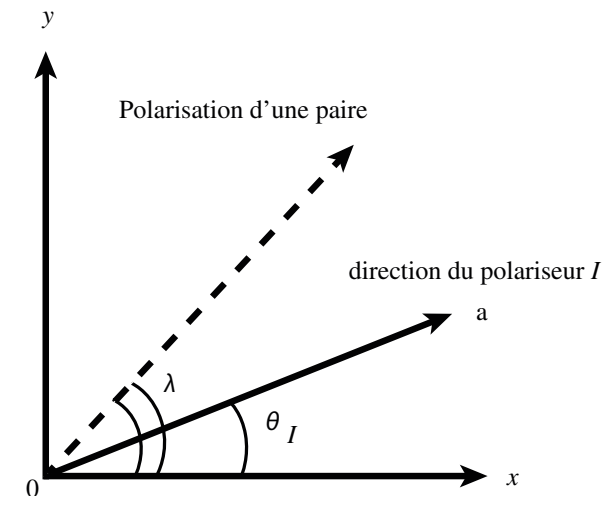

Figure 2. Exemple de théorie à paramètres supplémentaires. Chaque paire de photons a une "direction de polarisation", définie par $\lambda$, qui est le paramètre supplémentaire du modèle.

Le modèle nous permet également de calculer la fonction de corrélation de polarisation, et nous trouvons à partir de l'équation (I2) :

$$
\begin{aligned}
& E(\mathbf{a}, \mathbf{b})=1-4 \frac{\left|\theta_{I}-\theta_{I I}\right|}{\pi}=1-4 \frac{|(\mathbf{a}, \mathbf{b})|}{\pi} \\
& \text { pour } \quad-\frac{\pi}{2} \leq \theta_{I}-\theta_{I I} \leq \frac{\pi}{2}
\end{aligned}
$$

Ce résultat est remarquable. Notons d'abord que $\mathrm{E}(\mathbf{a}, \mathbf{b})$ dépend seulement de l'angle relatif $(\mathbf{a}, \mathbf{b})$, entre les polariseurs, comme la prédiction de la mécanique quantique (6). De plus comme on le voit sur la figure 3 , il y a une différence modérée entre les prédictions du modèle simple à paramètre supplémentaire et les prédictions quantiques. Les prédictions sont en accord parfait pour les angles 0 et $\pi / 2$, c'est-à-dire dans les cas de corrélation totale. Ce résultat, obtenu avec un modèle à paramètre supplémentaire extrêmement simple, est très encourageant et l'on pourrait espérer qu'un modèle plus sophistiqué serait capable de réduire plus exactement les prédictions quantiques. La découverte de Bell est que la recherche de tel modèle est sans espoir, comme nous allons le montrer maintenant.

\subsection{Inégalités de Bell}

Il y a de nombreuses formes différentes, et des démonstrations variées, des inégalités de Bell. Nous donnons ici une démonstration très simple conduisant à une forme directement utilisable pour les expériences ${ }^{3}$.

(3) Il est important de faire la distinction entre les inégalités qui montrent une contradiction mathématique avec la mécanique quantique, mais sans possibilité d'un test expérimental avec des appareils (nécessairement) imparfaits, et des inégalités qui permettent un test expérimental dès lors que les imperfections expérimentales n'excèdent pas certaines limites. 


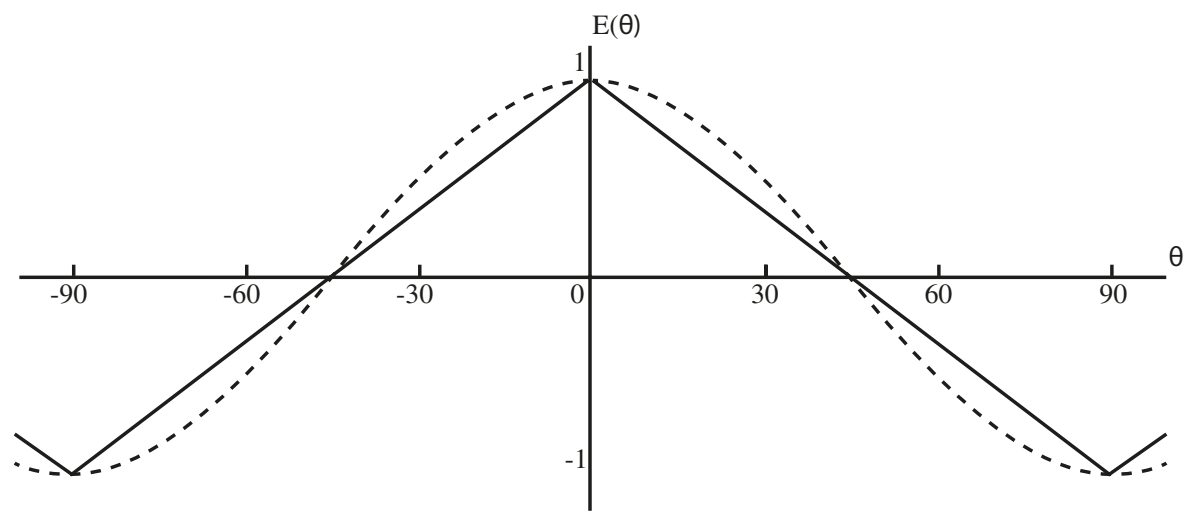

Figure 3. Coefficient de corrélation de polarisation, en fonction de l'orientation relative des polariseurs: (i) ligne pointillée : prédiction de la mécanique quantique ; (ii) ligne continue : notre modèle simple.

Considérons la quantité

$$
\begin{aligned}
s & =A(\lambda, \mathbf{a}) \cdot B(\lambda, \mathbf{b})-A(\lambda, \mathbf{a}) \cdot B\left(\lambda, \mathbf{b}^{\prime}\right)+A\left(\lambda, \mathbf{a}^{\prime}\right) \cdot B(\lambda, \mathbf{b})+A\left(\lambda, \mathbf{a}^{\prime}\right) \cdot B\left(\lambda, \mathbf{b}^{\prime}\right) \\
& =A(\lambda, \mathbf{a})\left[B(\lambda, \mathbf{b})-B\left(\lambda, \mathbf{b}^{\prime}\right)\right]+A\left(\lambda, \mathbf{a}^{\prime}\right)\left[B(\lambda, \mathbf{b})+B\left(\lambda, \mathbf{b}^{\prime}\right)\right]
\end{aligned}
$$

En se souvenant que les 4 nombres $A$ et $B$ ne peuvent prendre que les valeurs $\pm \mathrm{I}$, on voit immédiatement sur l'équation (17)

$$
s\left(\lambda, \mathbf{a}, \mathbf{a}^{\prime}, \mathbf{b}^{\prime}, \mathbf{b}^{\prime}\right)= \pm 2
$$

La moyenne sur $\lambda$ des quantités $s$ est donc comprise entre +2 et -2

$$
-2 \leq \int \mathrm{d} \lambda \rho(\lambda) \cdot s\left(\lambda, \mathbf{a}, \mathbf{a}^{\prime}, \mathbf{b}, \mathbf{b}^{\prime}\right) \leq 2
$$

En utilisant l'équation (12), nous récrivons ces inégalités

$$
-2 \leq S\left(\mathbf{a}, \mathbf{a}^{\prime}, \mathbf{b}, \mathbf{b}^{\prime}\right) \leq 2
$$

avec

$$
S\left(\mathbf{a}, \mathbf{a}^{\prime}, \mathbf{b}^{\prime}, \mathbf{b}^{\prime}\right)=E(\mathbf{a}, \mathbf{b})-E\left(\mathbf{a}, \mathbf{b}^{\prime}\right)+E\left(\mathbf{a}^{\prime}, \mathbf{b}\right)+E\left(\mathbf{a}^{\prime}, \mathbf{b}^{\prime}\right)
$$


Nous avons obtenu ainsi les inégalités B.C.H.S.H., qui sont les inégalités de Bell généralisées par Clauser, Horne, Shimony, Holt [7]. Elles portent sur une combinaison $S$ des quatre coefficients de corrélation associés à deux directions d'analyse pour chaque polariseur (a et a' pour le polariseur $I$, $\mathbf{b}$ et b' pour le polariseur $I I$ ).

\section{Conflit avec la mécanique quantique}

\section{I Mise en évidence}

Nous pouvons utiliser les prédictions (6) de la mécanique quantique pour les paires EPR, pour évaluer la quantité $S(\mathbf{a}, \mathbf{a}, \mathbf{b}, \mathbf{b}, \mathbf{b}$ ') définie par l'équation (2I). Pour les orientations particulières indiquées sur la figure $4 \mathrm{a}$, le résultat est

$$
S_{Q M}=2 \sqrt{2}
$$

Cette prédiction de la mécanique quantique est clairement en conflit avec les inégalités de Bell (20) qui sont valables pour n'importe quelle théorie à paramètre supplémentaire de la forme générale définie au §3.I.

Nous avons donc trouvé une situation où les prédictions de la mécanique quantique ne peuvent pas être reproduites (simulées) par des théories à paramètres supplémentaires. Ceci constitue l'essence du théorème de Bell : il est impossible de trouver une théorie à paramètres supplémentaires, de la forme générale définie au $\S 3.1$ qui reproduise toutes les prédictions de la mécanique quantique. Cette affirmation est la généralisation de ce que l'on voit sur la figure 3. Dans le cas particulier du modèle supplémentaire présenté au $\S 3.2$ : le modèle reproduit exactement les prédictions de la mécanique quantique pour certains angles $(0, \pi / 4, \pi / 2)$, mais il en diffère à d'autres angles. L'importance du théorème de Bell est qu'il n'est pas restreint à un modèle particulier mais qu'il est général.

\subsection{Conflit maximum}

Il est intéressant de chercher la violation maximale des inégalités de Bell par les prédictions quantiques. Considérons la valeur de $S$ prévue par la mécanique quantique :

$$
S_{Q M}\left(\mathbf{a}, \mathbf{b}, \mathbf{a}^{\prime}, \mathbf{b}^{\prime}\right)=\cos 2(\mathbf{a}, \mathbf{b})-\cos 2\left(\mathbf{a}, \mathbf{b}^{\prime}\right)+\cos \left(2 \mathbf{a}^{\prime}, \mathbf{b}\right)+\cos 2\left(\mathbf{a}^{\prime}, \mathbf{b}^{\prime}\right)
$$

Cette quantité est fonction des 3 variables indépendantes $(\mathbf{a}, \mathbf{b}),\left(\mathbf{b}, \mathbf{a}^{\prime}\right)$ et $\left(\mathbf{a}^{\prime}, \mathbf{b}^{\prime}\right)$. Notons que $\left(\mathbf{a}, \mathbf{b}^{\prime}\right)=(\mathbf{a}, \mathbf{b})+\left(\mathbf{b}, \mathbf{a}^{\prime}\right)+\left(\mathbf{a}^{\prime}, \mathbf{b}^{\prime}\right)$. 

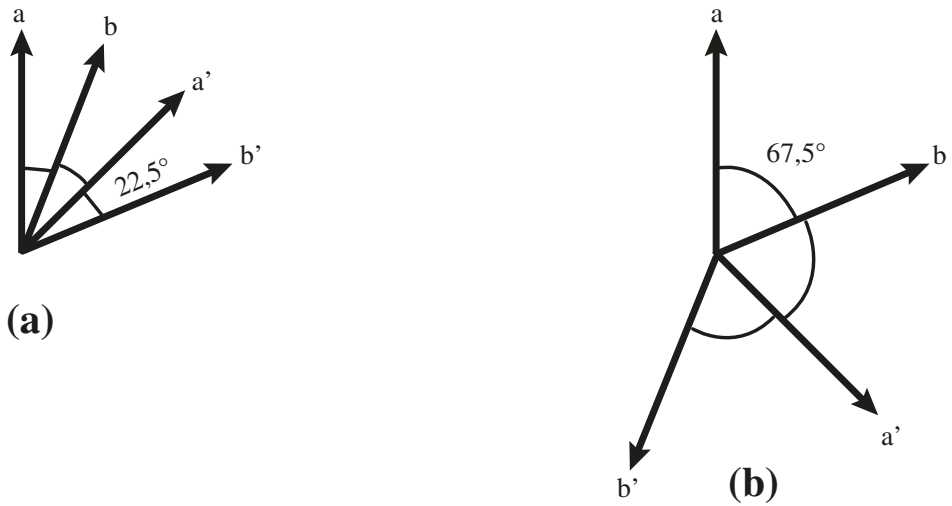

(a)

Figure 4. Orientations donnant le conflit maximum entre les inégalités de Bell et la mécanique quantique.

Afin de trouver les valeurs extrêmales de $S_{Q M}$, nous écrivons que les trois dérivées partielles sont nulles. On en déduit

$$
(\mathbf{a}, \mathbf{b})=\left(\mathbf{b}, \mathbf{a}^{\prime}\right)=\left(\mathbf{a}^{\prime}, \mathbf{b}^{\prime}\right)=\theta
$$

et

$$
\sin 2 \theta=\sin 6 \theta
$$

Nous avons tracé sur la figure 5 la fonction $S_{Q M}(\theta)$ évaluée avec des angles soumis à la contrainte (24). Ce graphe montre que les maximum et minimum absolus de $S_{Q M}$ sont

$$
\begin{aligned}
& S_{M Q}=2 \sqrt{2} \text { pour } \theta= \pm \frac{\pi}{8} \\
& S_{M Q}=-2 \sqrt{2} \text { pour } \theta= \pm \frac{3 \pi}{8}
\end{aligned}
$$

Ces valeurs sont bien solutions de (25). Les ensembles d'orientation correspondants sont indiqués sur la figure 4. Ils donnent les violations maximales des inégalités de Bell.

Plus généralement, la figure 5 montre qu'il y a un grand intervalle d'orientation conduisant à un conflit entre les prédictions quantiques et les inégalités de Bell. Cependant il est également clair que toutes les orientations ne donnent pas un tel conflit. 


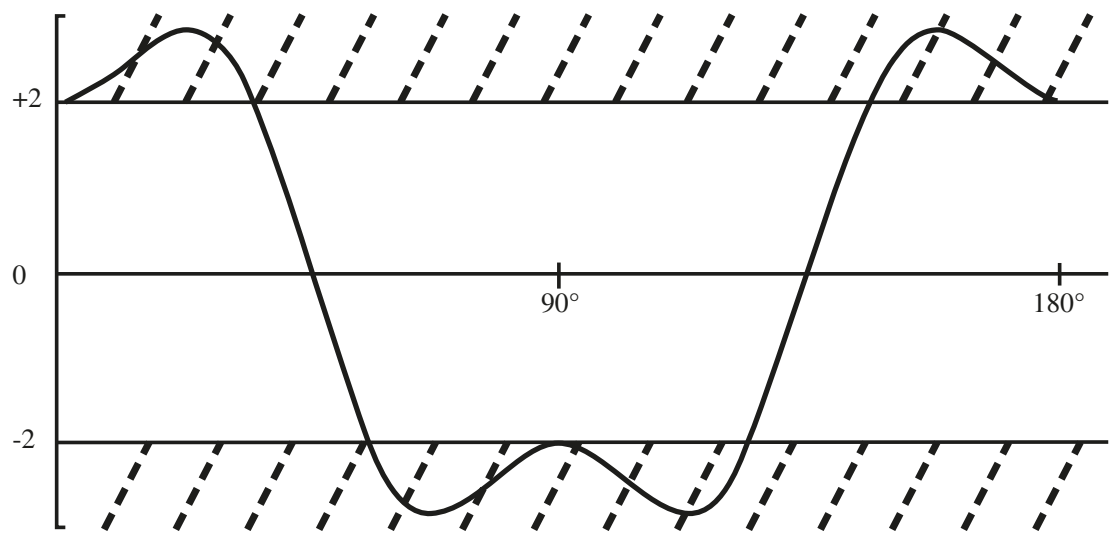

Figure 5. Quantité $S(\theta)$ prédite par la mécanique quantique pour des paires EPR. Le conflit avec les inégalités de Bell se produit quand $|S|$ est plus grand que 2 , et il est maximal pour les orientations indiquées sur la figure 4.

\section{Discussion : la condition de localité de Bell}

A ce point du raisonnement, nous avons établi le théorème de Bell : la mécanique quantique est en conflit avec n'importe quelle théorie à paramètres supplémentaires telle que nous l'avons définie au $\S 3.1$; en effet, la mécanique quantique viole les inégalités de Bell, mais ces inégalités s'appliquent automatiquement à toutes les théories à paramètres supplémentaires du type défini au § 3.1 . Il est intéressant de regarder en détail les hypothèses qui sous-tendent ce formalisme introduit au $\S 3$.I, dans le but de mettre en évidence une hypothèse particulière responsable du conflit.

Dans le paragraphe 3.I, on fait d'abord l'hypothèse de l'existence de paramètres supplémentaires. Comme nous l'avons vu, ces paramètres sont introduits pour rendre compte des corrélations à distance. Cette hypothèse est étroitement reliée à une conception du monde, défendue par Einstein, où la notion de réalités physiques séparées pour des systèmes séparés a une signification. Il est en fait possible de déduire l'existence de paramètres supplémentaires à partir de définitions très générales sur la réalité physique, dans l'esprit des idées d'Einstein [8]. Quel que soit le point de départ, une hypothèse dans cet esprit semble absolument nécessaire pour obtenir des inégalités en conflit avec la mécanique quantique.

La seconde hypothèse repose sur la remarque que le formalisme de la section 3.1 est déterministe : une fois que $\lambda$ est fixé, les résultats $A(\lambda, \mathbf{a})$ et $B(\lambda, \mathbf{b})$ des mesures de polarisation sont certains. On a suggéré que ce caractère déterministe pourrait être une raison cruciale du conflit avec le formalisme non déterministe de la mécanique quantique. En fait, comme John Bell [9], et d'autres l'ont montré [10], il est facile de généraliser le formalisme de la partie 3 .I à des théories à paramètres supplémentaires stochastiques, où les fonctions déterministes $A(\lambda, \mathbf{a})$ et $B\left(\lambda, \mathbf{b}^{\prime}\right)$ sont remplacées par des fonctions aléatoires. On trouve alors qu'il y a encore des inégalités de Bell, et que le conflit avec la mécanique quantique demeure. On peut en conclure que le caractère déterministe du formalisme n'est pas la raison du conflit [II]. 
Finalement, une troisième hypothèse implicite a été faite, comme l'a soulignée J. Bell dans tous ses articles : il s'agit de l'hypothèse de localité. Remarquons en effet que, dans le formalisme de la partie 3.I, nous avons supposé implicitement que le résultat $A(\lambda, \mathbf{a})$ de la mesure par le polariseur $I$ ne dépend pas de l'orientation $\mathbf{b}$ du polariseur éloigné $I I$, et vice-versa. De même, on suppose que la densité de probabilité $\rho(\lambda)$ (qui décrit la façon dont les paires sont émises) ne dépend pas des orientations $\mathbf{a}$ et $\mathbf{b}$. Cette condition de localité est cruciale : les inégalités de Bell ne s'appliqueraient pas à un formalisme ne le respectant pas. On constate en effet sans difficulté que la démonstration du $\S 3.3$ n'est plus valable avec des fonctions telles que $A(\lambda, \mathbf{a}, \mathbf{b})$ ou $\rho(\lambda, \mathbf{a}, \mathbf{b})$.

En conclusion, deux hypothèses semblent absolument nécessaires pour obtenir les inégalités de Bell et donc un conflit avec la mécanique quantique :

- Les corrélations à distance peuvent s'interpréter en introduisant des Paramètres Supplémentaires associés à chacune des deux particules séparées, dans l'esprit de l'idée défendue par Einstein que des objets séparés ont des réalités physiques séparées.

- Les quantités $A(\lambda), B(\lambda)$, et $\rho(\lambda)$ obéissent à la condition de localité, qui stipule qu'elles ne dépendent pas des orientations des polariseurs éloignés.

L'ensemble de ces deux hypothèses constitue ce que l'on appelle parfois la séparabilité d'Einstein. Comme ces deux hypothèses conduisent à un conflit avec la mécanique quantique, on en conclut que la mécanique quantique est non séparable.

On pourrait se demander laquelle de ces deux hypothèses est plus particulièrement responsable du conflit. En fait, il semble difficile de garder l'une sans l'autre. Cela a-t-il un sens de considérer un système séparé dans l'espace temps caractérisé par des propriétés propres, si ce système est en interaction non locale avec des systèmes éloignés ? II nous semble donc que l'idée même de système séparé est indissociable de celle de localité, et il ne nous paraît pas illégitime de confondre séparabilité d'Einstein et localité. C'est en ce sens que l'on pourra dire que la mécanique quantique est non locale.

\section{Expérience de pensée avec des polariseurs variables : la condition de localité comme conséquence de la causalité d'Einstein}

Dans des expériences statiques, où les polariseurs sont fixes pendant toute la durée d'une expérience, la condition de localité doit être posée comme une hypothèse. Bien qu'extrêmement raisonnable, cette condition n'est prescrite par aucune loi physique fondamentale. Comme l'écrit J. Bell [2] « les réglages des instruments sont faits suffisamment à l'avance pour leur permettre d'atteindre des états coordonnés par échange de signaux se produisant à une vitesse inférieure ou égale à celle de la lumière $»$. Dans ce cas, le résultat $A(\lambda)$ de la 
mesure au polariseur I pourrait dépendre de l'orientation b du polariseur éloigné $I$, et vice-versa. La Condition de Localité ne s'appliquerait alors plus, ni les inégalités de Bell.

Après cette remarque, Bell insiste sur l'importance des « expériences du genre proposées par Bohm et Aharonov [4], dans laquelle les réglagles sont modifiés pendant la propagation des particules $\aleph^{4}$. Dans une telle expérience dépendant du temps, la condition de localité deviendrait une conséquence de la causalité d'Einstein, qui interdit toute influence plus rapide que la lumière.

Comme nous l'avons montré dans notre propositon de 1975 [12], il suffit en fait, pour être dans un tel schéma, de commuter rapidement chaque polariseur entre deux orientations particulières (a et $\mathbf{a}^{\prime}$ pour $I$, $\mathbf{b}$ et b' pour $I I$ ). Il devient alors possible de tester expérimentalement une classe très vaste de théories à paramètres supplémentaires : toutes celles qui obéissent à la causalité d'Einstein. Dans de telles théories, la réponse du polariseur $I$ à l'instant $t$ peut dépendre de l'orientation b (ou b') du polariseur II, mais seulement aux instants antérieurs à $t-L / c$ ( $L$ est la distance entre les polariseurs). Une dépendance retardée analogue est prise en compte pour la densité de probabilité $\rho(\lambda)$, qui décrit l'émission des paires par la source. Pour des instants de commutation aléatoire, sans corrélation mutuelle entre les deux côtés éloignés, les prédictions de ces théories générales appelées «théories séparables à paramètres supplementaires », sont contraintes par des inégalités de Bell généralisées, basées sur la causalité d'Einstein et non plus sur la condition de localité de Bell [12].

Par ailleurs, on peut montrer [13] que les corrélations de polarisation prédites par la mécanique quantique dépendent seulement des orientations $\mathbf{a}$ et $\mathbf{b}$ à l'instant précis de la mesure et ne mettent en jeu aucun terme de retard $L / c$. Pour un choix convenable d'un ensemble d'orientations ( $\left.\mathbf{a}, \mathbf{a}^{\prime}, \mathbf{b}, \mathbf{b}^{\prime}\right)$ - par exemple les ensembles indiqués dans la figure 4 les prédictions de la mécanique quantique sont encore en conflit avec les inégalités de Bell généralisées.

Dans une expérience avec des polariseurs variables, le théorème de Bell établit donc l'incompatibilité entre la mécanique quantique et toutes les théories à paramètres supplémentaires obéissant à la causalité d'Einstein. Remarquons à nouveau ici que la causalité d'Einstein jouait déjà un rôle central dans les discussions conduisant à la notion de paramètres supplémentaires, ou de façon équivalente à l'existence d'une réalité physique indépendante pour chaque sous système séparé [5]. II ne semble donc pas exagéré de conclure que dans une expérience avec des polariseurs variables, le théorème de Bell établit une contradiction entre la mécanique quantique et une description du monde dans l'esprit des idées d'Einstein. Insistons cependant sur le fait qu'Einstein ne connaissait pas le théorème de Bell, et pouvait logiquement penser que sa conception du monde était compatible avec toutes les prédictions mathématiques de la mécanique quantique. Nous ne pouvons donc pas savoir quelle aurait été sa réaction devant la contradiction révélée par les inégalités de Bell.

(4) Cette idée était déjà indiquée dans le livre de Bohm [3]. 


\section{Du théorème de Bell à une expérience réelle}

\section{I Test expérimental des inégalités de Bell}

Après le théorème de Bell, le débat sur la possibilité (ou la nécessité) de compléter la mécanique quantique avait changé radicalement. Ce n'était plus une question de position philosophique (réalisme contre positivisme) ni de goût personnel. II devenait possible de trancher la question par une expérience.

En effet, si l'on peut produire des paires de photons (ou de particules de spin 1/2) dans un état EPR, et si l'on mesure les 4 taux de coïncidence $N_{ \pm \pm}(\mathbf{a}, \mathbf{b})$ avec des détecteurs placés dans les canaux de sortie des polariseurs (ou des filtres de Stern-Gerlach) on en tire directement le coefficient de corrélation de polarisation, pour des polariseurs dans les orientations $\mathbf{a}$ et $\mathbf{b}$ :

$$
E(\mathbf{a}, \mathbf{b})=\frac{N_{++}(\mathbf{a}, \mathbf{b})-N_{+-}(\mathbf{a}, \mathbf{b})-N_{-+}(\mathbf{a}, \mathbf{b})+N_{--}(\mathbf{a}, \mathbf{b})}{N_{++}(\mathbf{a}, \mathbf{b})+N_{+-}(\mathbf{a}, \mathbf{b})+N_{-+}(\mathbf{a}, \mathbf{b})+N_{--}(\mathbf{a}, \mathbf{b})}
$$

En répétant 4 mesures de ce type, dans les orientations (a,b), (a, $\left.\mathbf{b}^{\prime}\right),\left(\mathbf{a}^{\prime}, \mathbf{b}\right)$, et $\left(\mathbf{a}^{\prime}, \mathbf{b}^{\prime}\right)$, on obtient une valeur mesurée $S_{\text {exp }}\left(\mathbf{a}, \mathbf{a}^{\prime}, \mathbf{b}, \mathbf{b}^{\prime}\right)$ de la quantité $S$ définie par l'équation (2I). En choisissant une situation où la mécanique quantique prédit que ces quantités violent les inégalités de Bell (20), on a un test permettant de discriminer entre la mécanique quantique et l'ensemble des théories à paramètres supplémentaires locales. Si de plus on travaille dans un schéma avec des polariseurs variables, on teste la classe plus générale des théories à paramètres supplémentaires « séparables » (causales au sens relativiste).

\subsection{Les situations sensibles sont rares}

La mécanique quantique a été vérifiée dans tellement d'expériences que le théorème de Bell pourrait apparaître simplement comme une preuve de l'impossibilité des paramètres supplémentaires. En fait, les situations dans lequelles le conflit révélé par les inégalités de Bell se produit (situations « sensibles ») sont si rares que, en 1965, aucune n'avait été réalisée expérimentalement.

Pour mieux comprendre ce point, notons d'abord que les inégalités de Bell sont compatibles avec la totalité de la physique classique, c'est-à-dire la mécanique classique, (y compris relativiste), mais aussi l'électrodynamique classique. Il est en effet facile de montrer que ces théories classiques entrent dans le cadre des formalismes à paramètres supplémentaires obéissant à la causalité d'Einstein. Par exemple en mécanique classique, les paramètres $\lambda$ seraient les positions et les vitesses initiales des particules qui déterminent l'évolution future. De même en électrodynamique classique les $\lambda$ seraient les courants et les charges dans les sources, dont on peut déduire les champs électromagnétiques et leur action sur l'appareil de mesures. 
De plus, même dans les situations que l'on ne peut décrire que par la mécanique quantique, il n'y a que rarement un conflit avec les inégalités de Bell. Plus précisément lorsqu'on étudie les corrélations entre deux systèmes quantiques séparés (qui peuvent avoir interagi dans le passé), nous pouvons identifier deux conditions nécessaires pour avoir un conflit avec les inégalités de Bell :

- Les deux sous-systèmes séparés doivent être dans un état non-factorisable (état intriqué) analogue à (I) (ou à l'état singulet dans le cas de deux spin I/2).

- Pour chaque sous-système, il doit être possible de choisir la quantité mesurée parmi au moins deux observables qui ne commutent pas (comme par exemple des mesures de polarisation suivant deux directions $\mathbf{a}$ and $\mathbf{a}$ ', qui ne sont ni parallèles ni perpendiculaires).

Mais dans de telles situations, nous avons vu que le conflit n'existe que pour des quantités bien choisies (jeux d'orientations particuliers). Comme on le voit sur la figure 5 , il y a beaucoup d'orientations pour lesquelles les prédictions quantiques ne violent pas les inégalités de Bell.

Les physiciens se rendirent compte en 1965 qu'il n'existait aucun résultat expérimental mettant en évidence une violation des inégalités de Bell. Comme ces inégalités sont obtenues à partir d'hypothèses extrêmement raisonnables, on pouvait imaginer que la violation des inégalités de Bell pointe du doigt une situation où la mécanique quantique est en défaut. II était donc tentant de concevoir et réaliser une expérience sensible, c'est-à-dire une expérience où les prédictions de la mécanique quantique violent de façon non ambiguë les inégalités de Bell. Une telle expérience devrait trancher de façon claire entre la mécanique quantique et les théories à paramètres supplémentaires obéissant à la condition de localité de Bell.

\subsection{Source de paires de photons EPR}

Dans leur article, C.H.S.H. [7] remarquèrent que les paires de photons émises dans certaines cascades radiatives atomiques sont de bons candidats pour un test sensible. Considérons par exemple une cascade atomique $J=0 \rightarrow J=1 \rightarrow J=0$ (figure 6). Supposons que nous sélectionnions, avec des filtres en longueur d'onde et des collimateurs, deux ondes planes de fréquences $v_{1}$ et $v_{2}$ se propageant dans des directions opposées suivant l'axe $\mathrm{O} z$ (figure 7).

On peut montrer, en invoquant les conservations de la parité et du moment cinétique, que la polarisation de la paire $\left(v_{1}, v_{2}\right)$ est décrite par le vecteur d'état :

$$
\left|\Psi\left(v_{1}, v_{2}\right)\right\rangle=\frac{1}{\sqrt{2}}[|R, R\rangle+|L, L\rangle]
$$

où $|R\rangle$ et $|L\rangle$ sont des états de polarisation circulaire droite et gauche. En décomposant $|R\rangle$ et $|L\rangle$ sur une base de polarisation linéaire, on obtient finalement l'état (I) 


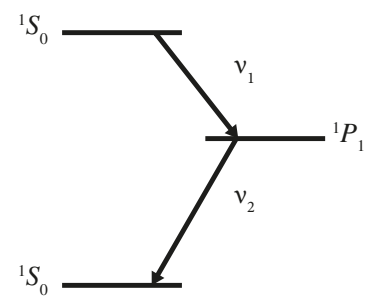

Figure 6. Cascade radiative émettant des paires de photons corrélés en polarisation.
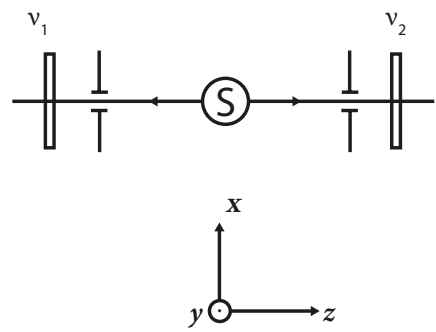

Figure 7. Configuration idéale (angles de détection infinitésimaux).

$$
\left|\Psi\left(v_{1}, v_{2}\right)\right\rangle=\frac{1}{\sqrt{2}}\{|x, x\rangle+|y, y\rangle\}
$$

Avec cet état EPR il est possible d'envisager une expérience sensible.

\subsection{Expérience réaliste}

Une expérience réelle diffère de l'expérience idéale à plusieurs égards. Par exemple la lumière est collectée dans un angle fini $2 u$, aussi grand que possible (figure 8). Dans cette situation, on peut montrer [14] que le contraste de la fonction de corrélation décroît, et l'équation (6) est remplacée par :

$$
E_{Q M}(\mathbf{a}, \mathbf{b})=F(u) \cdot \cos 2(\mathbf{a}, \mathbf{b})
$$

Où $F(u) \leq 1$

La figure 9 montre $F(u)$ pour une cascade radiative $J=0 \rightarrow J=1 \rightarrow J=0$ (atome alcalinoterreux sans structure hyperfine). On voit qu'il est possible d'utiliser de grands angles de corrélation sans perte notable. Ainsi pour $u=32^{\circ}$ (nos expériences), on a $F(u)=0,984$. 


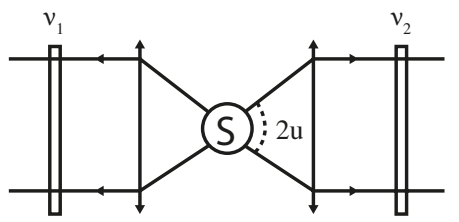

Figure 8. Configuration réaliste (angles de détection finis).

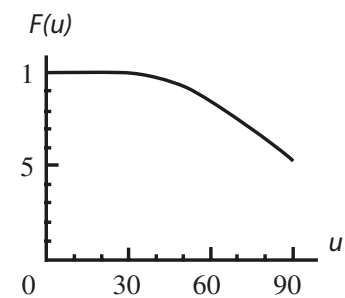

Figure 9. Facteur de réduction $F(u)$ pour une cascade $J=0 \rightarrow J=1 \rightarrow J=0$.

Tous les défauts expérimentaux (inefficacité des polariseurs, biréfringence fortuite, etc...) vont de façon analogue conduire à une diminution du contraste de la fonction de corrélation $E(\mathbf{a}, \mathbf{b})$. La fonction $S_{Q M}(\theta)$ (figure 5$)$ est donc multipliée par un facteur inférieur à I et le conflit avec les inégalités de Bell diminue, et peut même disparaître. On voit donc qu'une expérience réelle doit être conçue très soigneusement, et que tout effet annexe doit être évalué. Tous les paramètres importants doivent être contrôlés parfaitement car on peut imaginer qu'un effet oublié serait susceptible lui aussi de conduire à une diminution ou une disparition du conflit. Par exemple, on sait que la présence d'une structure hyperfine réduit considérablement $F(u)$, de telle sorte qu'il faut se limiter à des isotopes sans structure hyperfine [14].

\subsection{Conditions temporelles}

Nous avons indiqué dans la partie 6, que la condition de localité de Bell pouvait être considérée comme une conséquence de la causalité d'Einstein, si l'expérience remplit les conditions suivantes :

I. Les mesures distantes sur les deux sous-systèmes doivent être séparées par intervalle du genre espace.

2. Les choix entre les quantités mesurées sur les deux sous-systèmes séparés doivent être effectués aléatoirement, et être séparés par un intervalle du genre espace.

La seconde condition est manifestement plus difficile à remplir. 


\section{Expériences de première génération}

L'article C.H.S.H. [7], publié en 1969, montrait la possibilité d'expériences sensibles, avec des photons corrélés produits dans certaines cascades radiatives atomiques. Deux groupes lancèrent des expériences, une à Berkley, une à Harvard. Leurs résultats étaient contradictoires, et une troisième expérience fut montée à College Station (Texas). Ces trois expériences utilisaient un schéma expérimental simplifié, notablement différent du schéma réel car il utilisait des polariseurs à une voie.

\section{I Expérience avec des polariseurs à une voie}

Dans le schéma expérimental simplifié, on utilise des polariseurs qui transmettent la lumière polarisée parallèlement à $\mathbf{a}(\mathrm{ou} \mathbf{b})$, mais qui absorbent la polarisation orthogonale. Par rapport au schéma de la Figure I, on détecte donc seulement les résultats + , et les mesures de coïncidence fournissent seulement le taux de coïncidence $N_{++}(\mathbf{a}, \mathbf{b})$ entre les voies + . Pour suppléer aux données manquantes, on effectue des mesures complémentaires dans lesquelles un polariseur, ou l'autre, ou les deux sont effacés (par convention on note $\infty$ la situation où un polariseur est effacé). Nous pouvons écrire des relations entre les taux de coïncidences mesurés $N_{++}(\mathbf{a}, \mathbf{b}), N_{++}(\mathbf{a}, \infty)$ et $N_{++}(\infty, \mathbf{b})$ et des taux de coïncidence non mesurés :

$$
\begin{aligned}
& N_{++}(\infty, \infty)=N_{++}(\mathbf{a}, \mathbf{b})+N_{-+}(\mathbf{a}, \mathbf{b})+N_{-+}(\mathbf{a}, \mathbf{b})+N_{--}(\mathbf{a}, \mathbf{b}) \\
& N_{++}(\mathbf{a}, \infty)=N_{++}(\mathbf{a}, \mathbf{b})+N_{+-}(\mathbf{a}, \mathbf{b}) \\
& N_{++}(\infty, \mathbf{b})=N_{++}(\mathbf{a}, \mathbf{b})+N_{-+}(\mathbf{a}, \mathbf{b})
\end{aligned}
$$

En substituant ces relations dans l'expression (28) du coefficient de corrélation de polarisation, et dans les inégalités (2I), on peut éliminer toutes les quantités non mesurées et obtenir de nouvelles inégalités B.C.H.S.H. :

$$
-1 \leq S^{\prime} \leq 0
$$

où la quantité $S^{\prime}$

$$
S^{\prime}=\frac{N(\mathbf{a}, \mathbf{b})-N\left(\mathbf{a}, \mathbf{b}^{\prime}\right)+N\left(\mathbf{a}^{\prime}, \mathbf{b}\right)+N\left(\mathbf{a}^{\prime}, \mathbf{b}^{\prime}\right)-N\left(\mathbf{a}^{\prime}, \infty\right)-N(\infty, \mathbf{b})}{N(\infty, \infty)}
$$

s'exprime en fonction des seuls taux de coïncidence mesurée (nous avons omis les indices implicites ++ dans l'expression ci-dessus).

Pour les jeux d'orientation indiqués sur la figure 4, les prédictions de la mécanique quantique violent les inégalités de Bell (32), puisqu'elles donnent :

$$
\begin{array}{ll}
S_{Q M}^{\prime M a x}=\frac{\sqrt{2-1}}{2} & \text { pour } \quad \theta=\pi / 8 \\
S_{Q M}^{\prime M i n}=\frac{-\sqrt{2-1}}{2} & \text { pour } \theta=3 \pi / 8
\end{array}
$$


II est donc possible de faire un test sensible avec des polariseurs à une voie.

Notons cependant que la démonstration des inégalités de Bell modifiées (32) nécessite une hypothèse supplémentaire. En effet, les efficacités de détection étant faibles, à cause des ouvertures angulaires finies et des faibles efficacités de détection des photons, les probabilités apparaissant dans l'expression de $E(\mathbf{a}, \mathbf{b})$ doivent être redéfinies sur un ensemble restreint de paires, celles qui seraient détectées avec les polariseurs effacés. Cette procédure est valable seulement si on accepte une hypothèse raisonnable sur les détecteurs. Par exemple l'hypothèse de C.H.S.H. [7] admet que " pour une paire de photons émergeant du polariseur, la probabilité de détection en coïncidence est indépendante des orientations du polariseur » (ou du fait qu'ils sont effacés). Clauser et Horne [10] ont discuté une autre hypothèse, conduisant aux mêmes inénégalités. Le statut de ces hypothèses est discuté de façon approfondie dans la reference [15].

\subsection{Résultats}

Dans l'expérience de Berkeley [16], Clauser et Freedman construisirent une source où des atomes de calcium étaient excités par ultraviolet vers des états atomiques élevés. Parmi les diverses possibilités d'excitation, l'atome avait une certaine probabilité d'émettre une paire de photons verts ou violets (cascade radiative) $\left(4 p^{2}{ }^{1} S_{0} \rightarrow 4 s 4 p^{1} P_{1} \rightarrow 4 s^{2}{ }^{1} S_{0}\right)$. Dans cette expérience, le signal était particulièrement faible. De nombreuses cascades radiatives différentes de celles intéressantes se produisaient, et il fallut plus de $\mathbf{2 0 0}$ heures de mesures pour obtenir un résultat statistiquement significatif. Ce résultat fut trouvé en accord avec la mécanique quantique, et une violation des inégalités de Bell (32) fut observée (par 5 écarts type).

A la même époque, à Harvard, Holt et Pipkin [17] trouvèrent un résultat en désaccord avec la mécanique quantique et en accord avec les inégalités de Bell. Leur source de paires de photons corrélés reposait sur la cascade radiative $9^{1} P_{1} \rightarrow 7^{3} P_{1} \rightarrow 6^{3} P_{0}$ du mercure (isotope 200) excitée par bombardement électronique. Les données furent accumulées pendant 150 heures. Le même schéma expérimental fut repris quelques années plus tard par Clauser avec l'isotope 202 du mercure. Il trouva un résultat en accord avec la mécanique quantique conduisant à une violation significative des inégalités de Bell [18].

En 1976, à Texas A\&M, Fry et Thompson [19] construisirent une source de photons corrélés nettement plus efficace, basée sur la cascade radiative $7^{3} S_{1} \rightarrow 6^{3} P_{1} \rightarrow 6^{3} S_{0}$ du mercure 200. Ces auteurs pouvaient exciter sélectivement le niveau supérieur de la cascade, grâce à l'utilisation d'un laser continu monomode accordable (instrument exceptionnel à l'époque). Le signal était plus grand de plusieurs ordres de grandeurs, par rapport aux expériences précédentes, et il suffisait de 80 minutes d'accumulation de données pour obtenir des résultats significatifs. Ces résultats étaient en excellent accord avec la mécanique quantique, et on trouva une violation des inégalités de Bell (32) de quelques écarts types. 


\section{Les expériences d’Orsay (1980- 1982) [13]}

\section{I Source de phtotons corrélés}

Dès le début de notre programme en 1975 , notre but était de mettre en place des schémas expérimentaux plus sophistiqués que les précédents [12], et nous avons d'abord consacré de gros efforts au développement d'une source bien contrôlée de photons corrélés, de haute efficacité, et très stable. Ce résultat a été obtenu (figure 10) par une excitation sélective à deux photons [20] de la cascade radiative $4 p^{2}{ }^{1} S_{0} \rightarrow 4 s 4 p^{1} P_{1} \rightarrow 4 s^{2}{ }^{1} S_{0}$ du calcium déjà utilisée par Clauser et Freedman. Cette cascade est très bien adaptée aux exigences des mesures en coïncidence, car la durée de vie $\tau_{r}$ du niveau intermédiaire est relativement courte (5 ns). S'il est possible d'atteindre un taux d'excitation de l'ordre de $1 / \tau_{r}$, on obtient un rapport signalbruit optimal pour les mesures en coïncidence effectuées sur cette cascade.

Nous avons été capables d'atteindre ce taux optimal en utilisant deux lasers : un laser à krypton $\left(\lambda_{K}=406 \mathrm{~nm}\right)$ et un laser accordable $\left(\lambda_{D}=58 \mathrm{Imm}\right)$ réglés exactement à résonance pour le processeur à deux photons. Les deux lasers étaient monomodes. Ils étaient focalisés sur un jet atomique de calcium (sur environ $50 \mu \mathrm{m}$ ). Deux boucles d'asservissement permettaient d'obtenir une excellente stabilité de la source (meilleure que $0.5 \%$ pendant plusieurs heures) : la première boucle contrôle la longueur d'onde du laser accordable pour maximiser le signal de fluorescence ; la deuxième bouche contrôle l'angle entre les polarisations des lasers, ce qui permet d'ajuster le taux d'excitation pour compenser les fluctuations du taux d'émission des paires de photons. Il était possible avec quelques dizaines de milliwatts de chaque laser, d'obtenir un taux de cascades de $N=4 \times 10^{7} \mathrm{~s}^{-1}$. Une augmentation de ce taux au-delà de cette valeur n'aurait pas amélioré le rapport signal-bruit des comptages en coïncidence, car le taux des coïncidences fortuites croît comme $N^{2}$, tandis que le taux des coïncidences vraies croît comme $N$. Avec ce taux de cascade, le taux de coïncidence détecté avec des polariseurs parallèles était d'environ $10^{2} \mathrm{~s}^{-1}$, beaucoup plus grand que dans les expériences précédentes. Il était alors possible d'obtenir une précision statistique de I \% pour une durée de mesure 100 secondes seulement.

\subsection{Détection - Comptage en coïncidence}

La lumière de fluorescence était collectée par deux lentilles asphériques de grande ouverture (demi angle $u=32^{\circ}$, suivant la définition de la figure 8 ). Dans chaque voie de détection se trouvaient un filtre interférentiel (respectivement à $551,3 \mathrm{~nm}$ et $422,7 \mathrm{~nm}$ ), un système de transport optique, un polariseur, et un tube photomultiplicateur. Les sorties des photomultiplicateurs étaient connectées à une électronique de comptage en coïncidence, comportant un convertisseur temps-amplitude et un analyseur multicanal. On obtient ainsi le « spectre temps » des détections en coïncidence (figure II). Ce spectre comporte un fonds plat dû aux coïncidences fortuites (entre deux photons émis par des atomes distincts), tandis que les coïncidences vraies (entre les photons émis par le même atome) apparaîssent comme un pic montant brutalement à l'origine, et décroissant exponentiellement avec une constante de temps $\tau_{r}=5 \mathrm{~ns}$ (durée de vie du niveau intermédiaire de la cascade). Le signal de coïncidence vrai est l'aire de ce pic. 


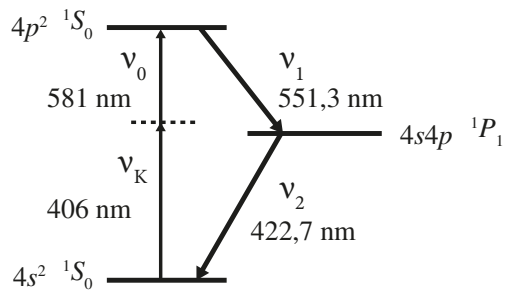

Figure 10. Excitation sélective à deux photons de la cascade radiative du calcium fournissant des paires de photons corrélés.

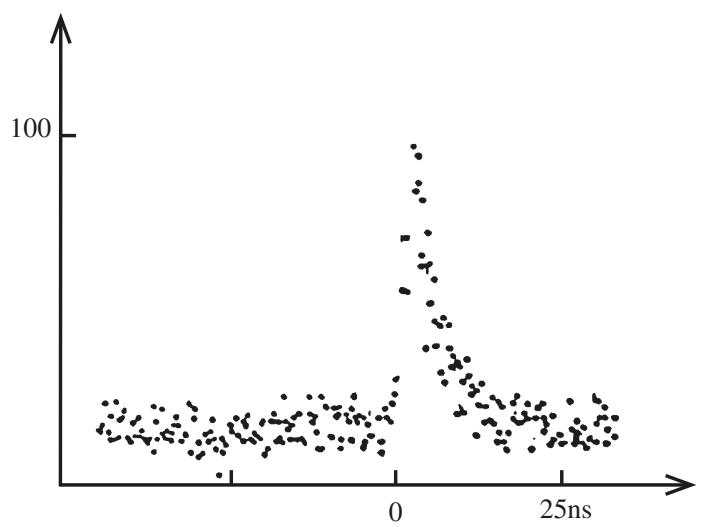

Figure 11. Spectre temps : nombre de paires détectées en fonction du retard entre les instants de détection des deux photons.

En parallèle un circuit de coïncidence standard, ayant une fenêtre de coïncidence de 19 ns, enregistre le taux de coïncidences autour du retard nul, tandis qu'un deuxième circuit avec un grand retard sur l'une des voies d'entrée enregistre le taux de coïncidences fortuites. Il est alors possible de vérifier que le taux de coïncidences obtenu par soustraction est égal à l'aire du pic du spectre-temps.

Dans les deuxième et troisième séries d'expériences décrites ci-dessous, nous avons utilisé un quadruple système de coïncidence, comportant un quadruple analyseur multicanaux et quatre doubles circuits de coïncidence standards. L'ensemble des données était collecté et traité automatiquement par ordinateur. 


\subsection{Expérience avec polariseurs à une voie [2I]}

Nos premières expériences furent réalisées avec des polariseurs à une voie. Il s'agissait de polariseurs à " pile de glace », comportant dix lames de verre de qualité optique à l'angle de Brewster, montées dans une mécanique de précision garantissant une excellente invariance par rotation. Pour de la lumière parfaitement polarisée, les transmissions maximale et minimale de ces polariseurs à une voie valaient respectivement 0,975 $\pm 0,005$ et $0,030 \pm 0,005$.

Grâce à notre source de haute efficacité, les incertitudes statistiques étaient de l'ordre de $1 \%$ dans chaque acquisition de $100 \mathrm{~s}$. Ceci nous permit de réaliser de nombreux tests statistiques et vérifications expérimentales, par exemple sur l'invariance par rotation des signaux (pour toutes ces mesures, la stabilité à long terme de la source, de l'ordre de $0.5 \%$, se révéla cruciale).

Un test direct des inégalités de Bell (32) adapté au cas des polariseurs à une voie, fut réalisé, on trouva pour la quantité $S$ ' (équation 33)

$$
S_{\exp }^{\prime}=0.126 \pm 0.014
$$

Ce résultat viole les inégalités (32) par 9 écarts type, et il est en bon accord avec les prédictions quantiques prenant en compte l'efficacité finie de nos polariseurs et l'ouverture des lentilles :

$$
S_{Q M}^{\prime}=0.118 \pm 0.005
$$

L'incertitude indiquée dans $S_{Q M}$, tient compte de l'incertitude sur les mesures des coefficients de transmission des polariseurs.

L'accord entre les données expérimentales et les prédictions de la mécanique quantique a pu être vérifié dans un intervalle d'orientations de $360^{\circ}$ (figure 12).

Ces expériences furent répétées avec des polariseurs éloignés de la source, chacun à une distance de $6,5 \mathrm{~m}$. A une telle distance (4 longueurs de cohérence associées à la durée de vie $t_{r}$ ) les détections sont des évènements séparés par un intervalle du genre espace, et nous remplissons donc la première condition temporelle indiquée au paragraphe 7.5. Dans cette nouvelle configuration, on n'a observé aucune modification des taux de coïncidence, et on a trouvé une violation des inégalités de Bell par la même quantité.

\subsection{Expérience avec polariseurs à deux voies [22-24]}

Avec des polariseurs à une seule voie, les mesures de polarisation sont par nature incomplètes. Lorsqu'une paire a été émise et qu'aucune détection n'est observée à l'un des 


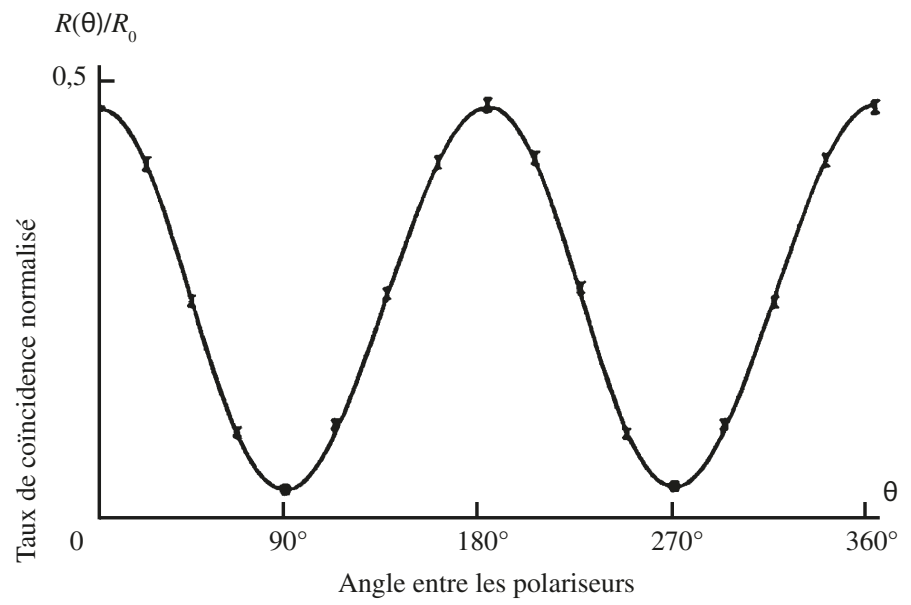

Figure 12. Expérience avec polariseurs à une voie. On indique le taux normalisé de coüncidences en fonction de l'orientation relative des polariseurs. Les incertitudes indiquées sont \pm 1 écart type. La courbe obtenue n'est pas un ajustement aux données, mais la prédiction de la mécanique quantique sans aucun paramètre ajustable.

détecteurs, il n'y a aucun moyen de savoir si la détection a été manquée par le détecteur ou si le photon a été arrêté par le polariseur (ce dernier cas correspondant à un résultat - pour la mesure). C'est pourquoi il faut utiliser des expériences auxiliaires, et un raisonnement indirect utilisant des hypothèses supplémentaires, pour pouvoir réaliser un test des inégalités de Bell adapté aux polariseurs à une seule voie.

Avec des polariseurs à deux voies, il devient possible de suivre beaucoup plus étroitement le schéma idéal de la figure I [22, 23,25]. Nous avons mené à bien une telle expérience, en utilisant des cubes polariseurs basés sur des multicouches diélectriques qui transmettent une polarisation et qui réfléchissent la polarisation orthogonale. Un séparateur de polarisation de ce type et deux photomultiplicateurs placés dans les deux voies de sortie, sont fixés dans une monture orientable. L'ensemble est un polarimètre qui fournit le résultat + ou le résultat - pour une mesure de polarisation linéaire suivant la direction choisie par l'expérimentateur. II s'agit d'un analogue optique d'un analyseur de Stern-Gerlach pour une particule à spin $\mathrm{I} / 2$.

A l'aide de deux polarimètres $I$ et $I I$ orientés suivant $\mathbf{a}$ et $\mathbf{b}$, et grâce à un quadruple système de comptage en coïncidence, nous sommes capables de mesurer en une seule période d'acquisition les quatre taux de coïncidence $N_{++}(\mathbf{a}, \mathbf{b})$, et d'en déduire directement le coefficient de corrélation de polarisation $E(\mathbf{a}, \mathbf{b})$ (cf. équation 28). Il est alors suffisant de refaire une mesure analogue pour trois autres orientations, et les inégalités de Bell (20) peuvent être testées diectement. 
Ce schéma expérimental est beaucoup plus proche du schéma idéal de la figure I que les expériences prédédentes avec des polariseurs à une voie, et en particulier il n'est pas nécessaire d'invoquer certaines hypothèses supplémentaires sur les détecteurs. Cependant, il faut noter que l'on est loin de détecter tous les photons, car l'efficacité de détection dans chaque voie est très inférieure à l'unité, d'abord à cause de l'angle solide de détection, et ensuite à cause du rendement quantique du photomultiplicateur. Un avocat des théories variables cachées pourrait alors soutenir que nous ne sommes pas sûrs que l'échantillon sur lequel porte la mesure reste le même lorsqu'on change les orientations. En toute logique, pour pouvoir confronter les mesures aux inégalités de Bell, il faut donc une hypothèse supplémentaire : on doit supposer que l'ensemble des paires effectivement détectées est indépendant de l'orientation des polariseurs.

Cette hypothèse est très raisonnable dans notre schéma expérimental symétrique, où les deux canaux de sorties d'un polariseur sont traitées sur le même pied (les efficacités de détection dans les deux canaux d'un polarimètre sont égales). De plus, nous avons vérifié expérimentalement que la somme des quatre taux de coïncidence $N_{ \pm \pm}(\mathbf{a}, \mathbf{b})$ reste constante lorsque les orientations changent, et ceci alors que chaque taux de coïncidence individuel est modulé à $100 \%$ : ceci prouve que la taille de l'échantillon sélectionné est constante. Evidemment, cela ne démontre pas de façon irréfutable la validité de l'hypothèse, mais au moins ces résultats ne contredisent pas cette hypothèse. Notons que l'on peut invoquer une hypothèse plus forte, mais plus facile à présenter : il s'agit de « l'hypothèse d'échantillonnage non biaisé », qui consiste à admettre que l'ensemble des paires détectées est un échantillon non biaisé représentatif de l'ensemble de toutes les paires émises. L'hypothèse envisagée plus haut est manifestement une conséquence de cette hypothèse plus forte.

Nous avons fait l'expérience décrite ci-dessus, dans les orientations de la figure 4a, pour lesquelles on prédit un conflit maximal. Nous avons trouvé

$$
S_{\text {exp }}=2.697 \pm 0.015
$$

ce qui viole les inégalités $(2 I)(|S| \leq 2)$ par plus de 40 écarts type. Notons de plus que le résultat est en parfait accord avec les prédictions de la mécanique quantique qui sont, compte tenu des imperfections des polariseurs et de l'ouverture des lentilles :

$$
S_{Q M}=2.70 \pm 0.05
$$

L'incertitude indiquée sur $S_{Q M}$ prend en compte une très légère dissymétrie des deux voies de sortie d'un polariseur $( \pm 1 \%)$. Nous avons calculé l'effet de ces dissymétries qui ne peuvent pas donner une variation de $S_{Q M}$ supérieure à $2 \%$.

Nous avons répété les mesures des coefficients de corrélation de polarisation $E(\mathbf{a}, \mathbf{b})$ à diverses orientations, et effectué une comparaison directe avec les prédictions de la mécanique quantique (figure 13). L'accord est manifestement excellent. 


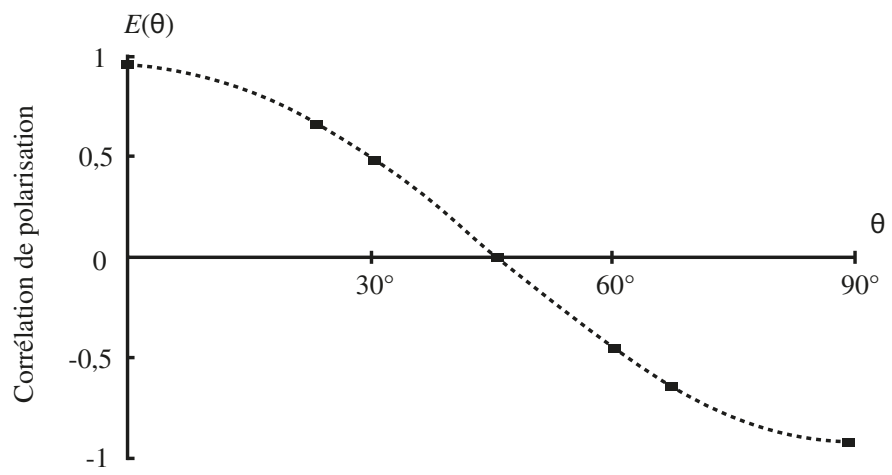

Orientation relative des polariseurs

Figure 13. Expérience avec polarisateurs à deux voies. Coefficient de corrélation de polarisation en fonction des orientations relatives des polarimètres. Les incertitudes portées sur la figure sont de \pm 2 écarts type. La courbe en pointillé n'est pas un ajustement aux données mais représente les prédictions de la mécanique quantique pour l'expérience réelle. Si l'expérience était parfaite, la courbe atteindrait les valeurs \pm 1 . On constate que l'expérience réelle est très voisine de l'expérience idéale.

\subsection{Expérience avec polariseurs variables [26]}

Comme nous l'avons souligné dans les parties 6 et 7.5, un test idéal des inégalités de Bell devrait offrir la possibilité d'un changement des orientations des polariseurs à des instants aléatoires [12]. Alors la condition de localité de Bell deviendrait une conséquence de la causalité d'Einstein. En 1982, nous avons fait un premier pas vers une telle expérience idéale en utilisant le schéma modifié indiqué sur la figure 14 .

Chaque polariseur (à une seule voie) est remplacé par un système comportant un commutateur suivi de deux polariseurs dans deux orientations : a et a' du côté $I, \mathbf{b}$ et b' du côté II. Chaque commutateur (aiguillage optique) est capable de diriger la lumière incidente soit vers un polariseur, soit vers l'autre, avec un temps de basculement très bref. Chaque dispositif est donc équivalent à un polariseur variable basculant d'une orientation à l'autre. Dans notre expérience, la distance $L$ entre les deux commutateurs vaut $13 \mathrm{~m}$, et $L / c$ vaut 43 ns.

Le basculement de la lumière s'effectue par interaction acousto-optique avec une onde stationnaire ultrasonique dans de l'eau. L'angle d'incidence (angle de Bragg) et la puissance acoustique sont réglés pour un basculement total entre les ordres de diffraction 0 et I. La fonction de commutation est donc de la forme $\sin ^{2}\left(\pi / 2 \cos \Omega_{a} t\right)$, la fréquence acoustique $\Omega_{a} / 2 \pi$ étant de l'ordre de $25 \mathrm{MHz}$. Les changements d'orientation équivalents se produisent donc au bout d'intervalles de temps de 6,7 ns et 13,3 ns. Comme ces intervalles entre deux commutations, aussi bien que l'intervalle de temps séparant les émissions des deux photons d'une même paire (valeur moyenne $\tau_{r}=5 \mathrm{~ns}$ ), sont petits devant $L / c$ (43 ns), l'événement constitué par la détection d'un côté est séparé par un intervalle du genre espace de l'événement 


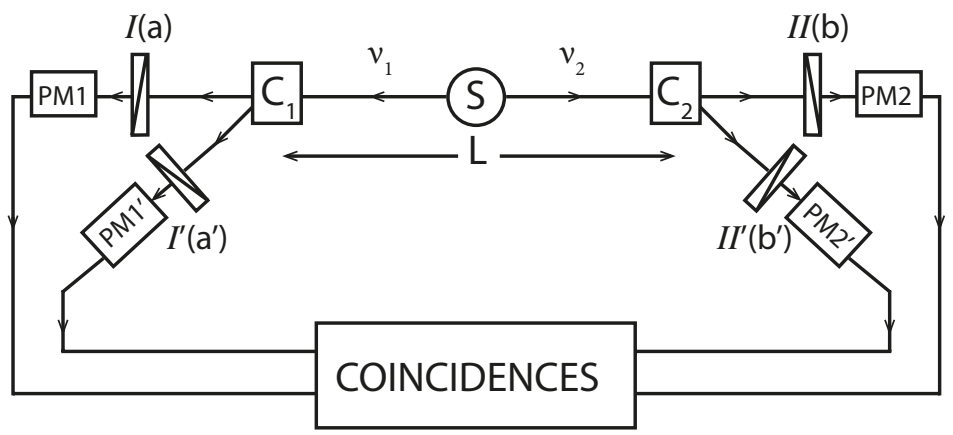

Figure 14. Expérience avec polarisateurs variables. Le commutateur $C_{1}$ dirige les photons $v_{1}$ soit vers le polariseur I soit vers I', dans les orientations a et a'. De même le commutateur $C_{2}$ dirige les photons $v_{2}$ soit vers le polariseur II soit vers II', dans les orientations $\mathbf{b}$ et $\mathbf{b}$ '. Les deux commutateurs sont pilotés par des générateurs indépendants. Les intervalles de temps entre les commutations sont respectivement de 6,7 ns et 13,3 ns.

associé au changement d'orientation correspondant de l'autre côté. La deuxième condition temporelle de la partie 5 est vérifiée, au moins partiellement. Nous notons en effet que la commutation était quasi périodique et non totalement aléatoire (voir discussion ci-dessous).

En fait, cette expérience de 1982 avec des polariseurs variables était loin d'être parfaite sur plusieurs autres points. D'abord, afin d'adapter les faisceaux de photons aux commutateurs, nous avons dû réduire leur taille par un facteur 3, entraînant une diminution du taux de coïncidence d'un ordre de grandeur. Dans ces conditions, pour obtenir une précision statistique suffisante, la durée de l'accumulation des données devait être notamment plus longue, et des problèmes de dérive sont apparus. II a donc fallu effectuer des moyennes des diverses quantités mesurées.

Par ailleurs, pour des faisceaux qui ne sont pas parfaitement collimatés, le contraste de la fonction de commutation n'est pas de 100\%, car l'angle d'incidence n'est pas exactement l'angle de Bragg pour tous les rayons. Dans notre expérience, le minimum de la fonction de commutation dans chaque canal valait $20 \%$ (au lieu de 0 dans le cas idéal).

Notons enfin que dans cette expérience nous avons utilisé des polariseurs à une seule voie; ce qui nous a permis de réaliser ces mesures avec le même système de coïncidence quadruple que l'expérience statique décrite au paragraphe 9.4.

Malgré toutes ces imperfections, cette expérience avec des polariseurs variables était la première à tester la non séparabilité quantique au sens d'Einstein. Le test des inégalités de Bell proprement dit porte sur des données accumulées pendant $8000 \mathrm{~s}$ avec 4 polariseurs dans les orientations de la figure 4.a. Par ailleurs, un total de $16000 \mathrm{~s}$ était consacré à des mesures avec une partie ou la totalité des polariseurs effacés. A cause des effets de dérives systématiques, les mesures étaient alternées toutes les $400 \mathrm{~s}$ entre les diverses orientations, et le résultat total résulte donc d'une moyenne. Nous avons ainsi obtenu 


$$
S_{\exp }^{\prime}=0.101 \pm 0.020
$$

ce qui viole la limite supérieure des inégalités de Bell (32) par 5 écarts type. Notons que ce résultat est en bon accord avec la prédiction quantique pour l'expérience réelle :

$$
S_{Q M}^{\prime}=0.113 \pm 0.005
$$

Nous avons effectué d'autres mesures des taux de coïncidences en fonction de l'orientation relative des polariseurs. La figure 15 montre que ces résultats sont en excellent accord avec la prédiction quantique.

A la lumière de ces résultats expérimentaux, les théories à paramètres supplémentaires obéissant à la causalité d'Einstein (théories à variables cachées séparables) semblent devoir être rejetées. Néanmoins, comme nous l'avons déjà mentionné, notre expérience est loin d'être idéale sur plusieurs points, et il reste plusieurs échappatoires pour un avocat des théories à variables cachées. D'abord, étant donné que nous utilisons des polariseurs à une seule voie, l'expérience n'est significative que si l'on accepte des versions fortes de l'hypothèse d'échantillonnages non biaisés. Quant au caractère temporel de cette expérience, un avocat des théories à variables cachées peut tirer argument du fait que le commutateur n'était pas total. Notons cependant qu'une proportion élevée des paires $(80 \%)$ subit la commutation forcée. Si les inégalités de Bell s'appliquaient à ces paires, tandis que les autres suivraient les prédictions quantiques, on aurait dû observer une différence notable entre les résultats expérimentaux et les prédictions quantiques.

Le point le plus important, relatif au caractère temporel, est le fait que les commutateurs n'étaient pas réellement aléatoires, puisque les commutateurs acousto-optiques étaient pilotés par des générateurs périodiques. Notons néanmoins que les deux générateurs pilotant les deux commutateurs étaient totalement indépendants, et opéraient à des fréquences différentes $(23,1 \mathrm{MHz}$ et $24,2 \mathrm{MHz})$, avec des dérives en fréquence non corrélées. Notons en outre, qu'il existe un caractère aléatoire de l'intervalle de temps entre l'émission des 2 photons d'une seule paire (distribution exponentielle de constante de temps $\tau_{r}=5 \mathrm{~ns}$, comme on le voit sur la figure II), ce qui ajoute un caractère aléatoire aux instants de détection associés à l'un ou l'autre polariseur.

En conclusion, cette expérience qui est restée jusqu'en 1998 la seule mettant en jeu des modifications rapides des orientations des polariseurs, avait suffisamment d'imperfections pour ne pas exclure certains modèles à paramètres supplémentaires ad hoc respectant la causalité d'Einstein. Notons néanmoins que plusieurs modèles de ce type peuvent être éliminés par l'ensemble des résultats expérimentaux, qui sont non seulement la valeur de $S^{\prime}$ indiquée en (39), mais aussi de nombreuses autres données et en particulier des spectres temps qui ne montrent aucun accident observable sur la décroissance exponentielle; si les résultats de mesure étaient différents suivant qu'ils sont séparés par un intervalle du genre temps ou espace, cela aurait du se traduire par un accident sur les spectres temps. 


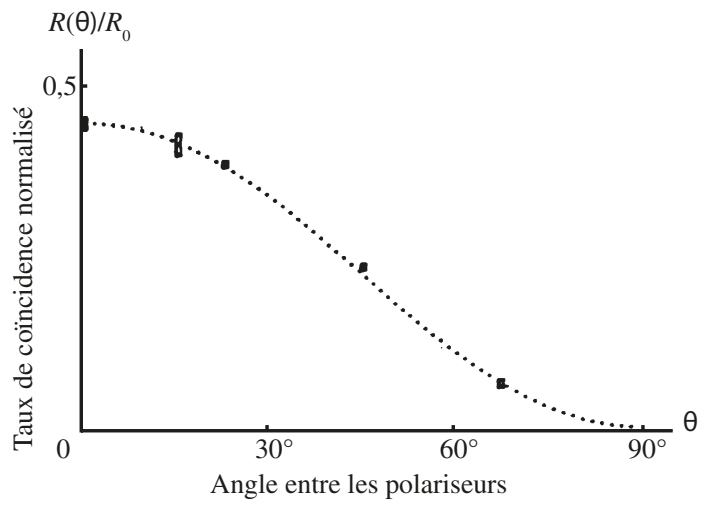

Figure 15. Expérience avec commutateur optique : taux de cö̈ncidence normalisés en fonction de l'orientation relative des polariseurs. Les incertitudes indiquées sont de \pm 1 écart type. La courbe en pointillés n'est pas un ajustement mais représente les prédictions de la mécanique quantique pour l'expérience réelle.

\section{Expérience de troisième génération : paire de photons produite par division paramétrique}

Comme nous l'avons déjà souligné, la cascade radiative du calcium utilisée dans nos expériences était excitée à un taux optimal au-delà duquel on ne peut plus améliorer le rapport signal à bruit. Sachant que cette cascade était a priori très favorable (à cause de la très courte durée de vie dans l'état intermédiaire), il ne restait guère de possibilités d'amélioration avec des sources utilisant des cascades radiatives atomiques [27].

Vers la fin des années 80 , un nouveau type de source de paires de photons corrélés a été développé simultanément par deux groupes $[28,29]$. Dans ces sources, une paire de photons rouges est produite par division paramérique d'un photon ultraviolet. Du fait de la condition d'accord de phase dans le cristal non linéaire utilisé, il y a une corrélation forte entre les directions d'émission des 2 photons d'une même paire et l'on peut donc en principe, par un filtrage spatial adapté, capturer avec certitude les 2 photons d'une même paire. Les taux de détection simple ou en coïncidence sont alors du même ordre de grandeur (au rendement quantique de détection près). Ceci est un progrès notable par rapport aux cascades radiatives atomiques dans lesquelles les deux photons d'une même paire sont émis dans des directions très peu corrélées [14] : le taux de coincidences est a priori dans ce cas $\Omega / 4 \pi$ fois plus faible que le taux détection simple (ici encore au rendement quantique de détection près). Le nouveau schéma permet de s'affranchir de ce facteur de réduction et ceci a des conséquences importantes, à la fois pratiques et fondamentales. Sur le plan pratique, on peut obtenir des taux de coïncidence beaucoup plus élevés : dans le cas le plus favorable [30] le taux de coïncidence est supérieur de plus d'un ordre de grandeur à celui observé dans nos meilleures expériences. De plus, les photons sont produits dans des faisceaux de petite étendue (petit nombre de Fresnel). De tels faisceaux peuvent être facilement injectés dans des composants optiques de petite taille, et même dans des fibres optiques, ce qui ouvre de nombreuses possibilités nouvelles. 
Ces nouvelles sources peuvent produire des paires de photons corrélés en polarisation [28-3I], dans des états analogues à celui décrit par l'équation (I). Mais on peut aussi produire des états intriqués donnant lieu à des corrélations EPR entre des observables autres que la polarisation. Une situation intéressante [32] porte sur des paires de photons dont les membres sont émis «à deux instants différents ». Dans ce cas, l'observable pertinente est l'instant d'émission des photons. Plusieurs expériences ont été réalisées avec ce schéma [33-35]. Notons qu'un tel schéma expérimental, où la polarisation n'est pas l'observable mesurée, est particulièrement intéressant pour des expériences avec des fibres optiques, où le contrôle de la polarisation est un point particulièrement délicat. Une autre expérience intéressante utilise comme observable les directions d'émission des photons [36] : chaque photon d'une paire implique la superposition de deux directions d'émission différentes, respectivement corrélés aux deux directions d'émission du second photon. Une expérience de ce type a été réalisée [37].

Comme le souligne la référence Horn et al. [37], toutes ces expériences peuvent être présentées dans le cadre général des " interférences à deux particules » : effectivement, les probabilités conjointes des résultats de mesure se présentent comme le carré de la somme de deux amplitudes (chacune de ces amplitudes implique les deux photons), dont la phase relative peut être contrôlée expérimentalement. En fait, le schéma original EPR est un exemple très clair de cette situation. Par exemple, dans la version présentée au paragraphe 2, l'état (I) peut être réécrit (cf. équation 29) comme la superposition d'un état $|L, L\rangle$ où les deux photons ont une hélicité gauche et d'un état $|R, R\rangle$ où les deux photons ont une hélicité droite. Pour chacun de ces deux états, l'amplitude quantique associée à la détection conjointe dans un couple donné de canaux de sortie (voir la figure I), a une phase qui dépend de l'orientation relative des polariseurs. L'addition des amplitudes associées respectivement à $|L, L\rangle$ et $|R, R\rangle$ conduit donc à une interférence responsable des variations sinusoïdales de la probabilité conjointe (cf. équation 3).

Ces nouvelles sources et ces nouveaux schémas ont conduit à une série de tests des inégalités de Bell qui ont tous confirmé la mécanique quantique. Des violations non ambigües des inégalités de Bell ont été observées, sous l'hypothèse de l'échantillonage non biaisé. Notons en particulier une violation des inégalités de Bell par 100 écarts type dans une expérience durant seulement quelques minutes [30].

On peut citer une série d'expériences mettant en jeu des fibres optiques, avec des bras de plus en plus longs entre source et détecteurs. Ainsi, une violation claire des inégalités de Bell a été observée alors qu'un des bras de l'appareil était constitué d'une fibre optique de $4 \mathrm{~km}$ de long [34]. Puis, les corrélations EPR ont été observées avec des photons se propageant dans plusieurs dizaines de kilomètres de fibres optiques pour télécommunications [38]. Mais surtout, ces expériences à fibres optiques ont ouvert la voie à une expérience avec polariseurs variables (paragraphes 7.5 et 9.5), quasiment idéale.

Cette expérience [39] a complètement pris en compte les conditions temporelles relativistes du paragraphe 7.5. Rappelons que dans le schéma idéal [12], il faut des polariseurs dont les orientations puissent être modifiées à des instants aléatoires avec un temps caractéristique de changement d'orientation plus court que le temps de propagation de la lumière $L / c$ entre les deux polariseurs. Avec les nouveaux schémas utilisant les fibres 
optiques, il est devenu possible de travailler avec des systèmes électrooptiques intégrés, à des distances de la source correspondant à des temps de propagation de plusieurs microsecondes. On peut alors, pendant ce temps propagation, effectuer un tirage aléatoire de l'orientation du polariseur, puis positionner la direction d'analyse suivant la direction choisie [39]. Ultime raffinement de cette expérience, les coïncidences ne seront dépouillées qu'après la fin de la période d'acquisition. Pendant l'acquisition proprement dite, on « se contente » en effet d'enregistrer à chaque polariseur l'ensemble des paramètres de chaque événement détecté : orientation du polariseur, résultat obtenu, instant de détection (mesuré avec une horloge atomique). C'est la comparaison a posteriori des évènements détectés aux deux extrémités qui permet de déterminer les corrélations. Le résultat a confirmé sans ambiguité la violation des inégalités de Bell, et donné un remarquable accord avec les prévisions quantiques.

A cause de la corrélation quasi parfaite entre les directions d'émission des photons, cette troisième génération d'expériences devrait également permettre de fermer l'échappatoire liée aux faibles efficacités de détection, lorsque des détecteurs avec des efficacités quantiques de l'ordre de l'unité deviendront disponibles, ce qui ne devrait guère tarder ${ }^{5}$.

\section{I. Conclusion}

Nous avons donc aujourd'hui une quantité impressionnante de résultats obtenus dans des schémas expérimentaux sensibles et qui donnent des violations non ambigües des inégalités de Bell. De plus, ces résultats sont en excellent accord avec des prédictions quantiques qui prennent en compte toutes les caractéristiques connues des expériences réelles. Bien que toutes les échappatoires n'aient pas encore été fermées, et donc que des expériences améliorées restent encore souhaitables, il est légitime de discuter les conséquences de la réjection des théories à paramètres supplémentaires obéissant à la causalité d'Einstein [40].

II semble possible de conclure qu'il est possible d'observer dans la nature la non localité quantique, au sens expliqué aux paragraphes 5 et 6 , et ceci sur des distances très grandes, à l'échelle du kilomètre. Notons cependant que cette non localité quantique est de nature très subtile, et par exemple qu'on ne peut pas l'utiliser pour une « télégraphie plus rapide que la lumière ». On peut en effet montrer [4I] que dans un schéma où l'on voudrait utiliser les corrélations EPR pour envoyer un message, il serait nécessaire d'envoyer aussi une information classique complémentaire transitant par un canal classique. Aucun message utilisable n'est donc disponible avant le délai relativiste precrit par la causalité d'Einstein. Cela rappelle les schémas de téléportation quantique [42], qui permettent de transporter un état quantique de façon non locale, mais en utilisant aussi une information classique passant par un canal classique. Il y a en fait beaucoup à apprendre, et à comprendre, sur la non localité quantique, par une analyse approfondie des schémas de téléportation quantique [43].

(5) De telles expériences ont effectivement été menées à bien en 2015, voir par exemple : Alain Aspect, Closing the door on the Einsteins and Bohr's Quantum Debate, Physics 8, 123 (20I5). Available at URL: http://link.aps.org/doi/l0.1/03/Physics.8.123. 
Chaque fois que l'on se replonge dans le problème que nous venons de présenter, on ne peut s'empêcher de se poser la question : y a-t-il un problème réel ? II faut reconnaître que la réponse à cette question peut varier, même pour les plus grands physiciens. En 1963, R. Feynman donnait une première réponse à cette question dans son fameux cours de physique [44] : «Ce point ne fut jamais accepté par Einstein... Il devint connu sous le nom de paradoxe d'Einstein-Podolsky-Rosen. Mais lorsque la situation est décrite comme nous l'avons fait ici, il ne semble pas y avoir quelque paradoxe que ce soit... ${ }^{6}$. Deux décennies plus tard, Feynman exprimait une opinion radicalement différente [45], toujours sur la situation EPR : «nous avons toujours eu une très grande difficulté à comprendre la vision du monde que la mécanique quantique implique ... II ne m'est pas encore apparu évident qu'il n'y ait pas de problème réel... Je me suis toujours illusionné moi même, en confinant les difficultés de la mécanique quantique dans un recoin de plus en plus petit, et je me retrouve de plus en plus chagriné par ce point particulier. II semble ridicule de pouvoir réduire ce point à une question numérique, le fait qu'une chose est plus grande qu'une autre chose. Mais voilà : - elle est plus grande ... ${ }^{7}$

\section{Que rajouter?}

\section{Références}

[I] A. Einstein, B. Podolsky and N. Rosen, Can Quantum-Mechanical description of physical reality be considered complete? Phys. Review 47, 777 (1935).

See also Bohr's answer: N. Bohr, Can Quantum-Mechanical description of physical reality be considered complete? Phys. Review 48, 696 (1935).

[2] J. S. Bell, On the Einstein-Podolsky-Rosen Paradox, Physics I, 195 (1964).

[3] D. Bohm, Quantum Theory, Prentice-Hall, Englewoods Cliffs (195I). Republished by Dover (1989).

[4] D. Bohm and Y. Aharonov, Discussion of Experimental Proof for the Paradox of Einstein, Rosen and Podolsky, Phys. Rev. 108, 1070 (1957).

[5] A. Einstein philosopher scientist, P.A. Schilp ed. (Open court and Cambridge university press, 1949).

[6] Correspondance entre A. Einstein and M. Born (traduction française : Seuil, Paris, 1972).

[7] J.F. Clauser, M.A. Horne, A. Shimony and R.A. Holt, Proposed experiment to test local hidden-variable theories, Phys. Rev. Lett. 23, 880 (1969).

[8] B. d'Espagnat, Use of inequalities for the experimental test of a general conception of the foundation of microphysics, Phys. Rev. DII, I424 (1975).

L. Hardy, Quantum Mechanics, Local Realistic Theories, and Lorentz-Invariant Realistic Theories, Phys. Rev. Lett. 68, 298I (1992).

(6) «This point was never accepted by Einstein... It became known as the Einstein-Podolsky-Rosen paradox. But when the situation is described as we have done it here, there doesn't seem to be any paradox at all... \ [44]

(7) «We always have had a great deal of difficulty in understanding the world view that quantum mechanics represents... It has not yet become obvious to me that there is no real problem... I have entertained myself always by squeezing the difficulty of quantum mechanics into a smaller and smaller place, so as to get more and more worried about this particular item. It seems almost ridiculous that you can squeeze it to a numerical question that one thing is bigger than another. But there you are - It is bigger... » [45]. 
[9] J.S. Bell, Introduction to the Hidden-Variable Question, in : « Foundations of Quantums Mechanics », B. d'Espagnat ed., Academic, N.Y. (1972).

[I0] J. F. Clauser and M. A. Horne, Experimental consequences of objective local theories, Phys. Rev. DI0, 526 (1974).

[II] Cette conclusion n'est pas partagée par tous les auteurs. Ainsi, on a pu écrire que les théories stochastiques à paramètres supplémentaires de Bell [9] ou de Clauser et Horne [10] ne sont pas vraiment plus générales, car on peut les reproduire (les simuler) par des théories déterministes: A. Fine, Hidden Variables, Joint Probability, and the Bell Inequalities, Phys. Rev. Lett. 48, 29I (1982).

[12] A. Aspect, Proposed Experiment to Test Separable Hidden-Variable Theories, Phys. Lett. 54A, 117 (1975).

A. Aspect, Proposed Experiment to test the nonseparability of Quantum Mechanics, Phys. Rev. DI4, 1944 (1976).

[13] A. Aspect, Trois tests expérimentaux des inégalités de Bell par mesure de corrélation de polarisation de photons, thèse d'Etat, Orsay (1983).

[14] E.S. Fry, Two-Photon Correlations in Atomic Transitions, Phys. Rev. A8, 1219 (1973).

[15] J.F. Clauser and A. Shimony, Bell's Theorem : Experimental Tests and Implications, Rep. Progr. Phys. 4I, I88I (1978).

[16] S.J. Freedman and J.F. Clauser, Experimental test of local hidden-variable theories, Phys. Rev. Lett. 28, 938 (1972).

[17] F.M. Pipkin, Atomic Physics Tests of the Basics Concepts in Quantum Mechanics, in : « Advances in Atomic and Molecular Physics », D.R. Bates fand B. Bederson, ed., Academic (1978).

[18] J.F. Clauser, Experimental Investigation of a Polarization Correlation Anomaly, Phys. Rev. Lett. 36, 1223 (1976).

[19] E.S. Fry, and R.C. Thompson, Experimental Test of Local Hidden-Variable Theories, Phys. Rev. Lett. 37, 465 (1976).

[20] A. Aspect, C. Imbert, and G. Roger, Absolute Measurement of an Atomic Cascade Rate Using a Two Photon Coincidence Technique. Application to the $4 p^{2} S_{0}-4 s 4 p ' P_{1}-4 s^{2} S_{0}$ Cascade of Calcium excited by a Two Photon Absorption, Opt. Comm. 34, 46 (1980).

[2I] A. Aspect, P. Grangier and G. Roger, Experimental Tests of Realistic Local Theories via Bell's Theorem, Phys. Rev. Lett. 47, 460 (198I).

[22] A. Aspect, P. Grangier and G. Roger, Experimental Realization of Einstein-Podolsky-RosenBohm Gedankenexperiment: A New Violation of Bell's Inequalities, Phys. Rev. Lett. 49, 91 (1982).

[23] P. Grangier, Thèse de troisième cycle, Orsay (1982).

[24] A. Aspect and P. Grangier, About Resonant Scattering and Other Hypothetical Effects in the Orsay Atomic-Cascade Experiment Tests of Bell Inequalities: A Discussion and some New Experimental Data, Lett. Nuovo Cimento 43, 345 (1985). 
[25] A. Garruccio and V. A. Rapisarda, Bell's inequalities and the four-coincidence experiment, Nuovo Cimento Al8, 269 (1982).

[26] A. Aspect, J. Dalibard and G. Roger, Experimental Test of Bell's Inequalities Using Variable Analyzers, Phys. Rev. Lett. 49, 1804 (1982).

[27] W. Perrie, A. J. Duncan, H. J. Beyer, and H. Kleinpoppen, Plarization Correlation of the Two Photons Emitted by Metastable Atomic Deuterium : A test of Bell's Inequality, Phys. Rev. Lett. 54, 1790 (1985) et 54, 2647 (E). Although the statistical significance of the results was limited as a test of Bell's inequalities, this experiment was a remarkable « tour de force » in atomic physics.

[28] Y. H. Shih and C. O. Alley, New type of Einstein-Podolsky-Rosen-Bohm Experiment using pairs of light quanta produced by optical parametric down conversion, Phys. rev. Lett. 61, 2921 (1988).

[29] Z.Y. Ou and L. Mandel, Violation of Bell's Inequality and Classical Probability in a Two-Photon Correlation Experiment, Phys. Rev. Lett. 61,50 (1988).

[30] P.G. Kwiat, K. Mattle, H. Weinfurter, and A. Zeilinger, New High-Intensity Source of Polarization-Entangled Photon-Pairs, Phys. Rev. Lett. 75, 4337 (1995).

Note that the reported violation of Bell's inequalities by 100 standard deviations relies on a stronger version of the « fair sampling hypothesis » than our second experiment (Section 9.4), since this experiment uses one channel polarizers and not two channel polarizers.

[3I] T.E. Kiess, Y.H. Shih, A.V. Sergienko, and C.O. Alley, Einstein-Podolsky-Rosen-Bohm Experiment Using Pairs of Light Quanta Produced by Type-Il Parametric Down-Conversion, 7I, 3893 (1993).

[32] J.D. Franson, Bell Inequality Position and Time, Phys. Rev. Lett. 62, 2205 (1989).

[33] J. Brendel, E. Mohler, and W. Martienssen, Experimental Test of Bell's Inequality for Energy and Time, Europhys. Lett. 20, 575.

[34] P.R.Tapster, J.G. Rarity, and P. C. M. Owens, Violation of Bell's Inequality over 4 km of Optical Fiber, Phys. Rev. Lett. 73, 1923 (1994).

[35] W. Tittel, J. Brendel, T. Herzog, H. Zbinden and N. Gisin, Non-local two-photon correlations using interferometers physically separated by 35 meters, Europhys., Lett. 40, 595 (1997).

[36] M.A. Horne, A. Shimony, A. Zeilinger, Two-Particle Interferometry, Phys. rev. Lett. 62 , 2209 (1989).

[37] J.G. Rarity and P.R. Tapster, Experimental Violation of Bell's Inequality Based on Phase and Momentum, Phys. Rev. Lett. 64, 2495 (1990).

[38] W. Tittel, J. Brendel, H. Zbinden, and N. Gisin, Violation of Bell's Inequalities by Photons more than 10 km Apart, Phys. Rev. Lett. 8I, 3563 (1998).

[39] Gregor Weihs, Thomas Jennewein, Christoph Simon, Harald Weinfurter, Anton Zeilinger, Violation of Bell's inequality under strict Einstein locality conditions, Phys. Rev.Lett. 8I 5039 (1998).

[40] J.S. Bell, Atomic cascade Photons and Quantum-Mechanical Nonlocality, Comments on Atom. Mol.Phys. 9, 121 (1981). 
[4I] A. Aspect, Expériences basées sur les inégalités de Bell, J. Physique Colloque C2, 940 (198I).

[42] C. H. Bennet, G. Brassard, C. Crépeau, R. Josza, A. Peres, and W. K. Wootters, Phys. Rev. Lett. 70, 1895 (1993).

D. Bouwmeester, J.-W. Pan, K. Mattle, M. Eibl, H. Weinfurter, and A. Zeilinger, Experimental quantum teleportation, Nature 390, 575 (1997).

D. Boschi, S. Branca, F. De Martini, L. Hardy, and S. Popescu, Experimental Realization of Teleporting an unknown pure Quantum State via Dual Classical and Einstein-Podolsky-Rosen Channels, submitted to Phys. Rev. Lett. (1997).

[43] S. Popescu, Bell's inequalities versus teleportation: what is non locality? Phys. Rev. Lett. 72, 797 (1994).

[44] The Feynman Lectures on Physics, vol. III, chapter 18.

[45] R. P. Feynman, Simulating Physics with Computers, Intern. Journ. of Theoret. Phys. 2I, 467 (1982). 